\title{
Monte Carlo control loops for cosmic shear cosmology with DES Year 1 data
}

T. Kacprzak $\odot,{ }^{1,}{ }^{*}$ J. Herbel, ${ }^{1}$ A. Nicola,${ }^{2}$ R. Sgier,${ }^{1}$ F. Tarsitano, ${ }^{1}$ C. Bruderer, ${ }^{1}$ A. Amara, ${ }^{1}$ A. Refregier, ${ }^{1}$ S. L. Bridle, ${ }^{3}$ A. Drlica-Wagner, ${ }^{4,5}$ D. Gruen, ${ }^{6,78}$ W. G. Hartley, ${ }^{9}$ B. Hoyle, ${ }^{10,11}$ L. F. Secco, ${ }^{12}$ J. Zuntz, ${ }^{13}$ J. Annis, ${ }^{4}$ S. Avila, ${ }^{14}$ E. Bertin, ${ }^{15,16}$ D. Brooks, ${ }^{9}$ E. Buckley-Geer, ${ }^{4}$ A. Carnero Rosell, ${ }^{17,18}$ M. Carrasco Kind, ${ }^{19,20}$ J. Carretero, ${ }^{21}$ L. N. da Costa, ${ }^{18,22}$ J. De Vicente, ${ }^{17}$ S. Desai, ${ }^{23}$ H. T. Diehl, ${ }^{4}$ P. Doel,${ }^{9}$ J. García-Bellido, ${ }^{14}$ E. Gaztanaga, ${ }^{24,25}$ R. A. Gruendl, ${ }^{19,20}$ J. Gschwend, ${ }^{18,22}$ G. Gutierrez, ${ }^{4}$ D. L. Hollowood, ${ }^{26}$ K. Honscheid, ${ }^{27,28}$ D. J. James, ${ }^{29}$ M. Jarvis, ${ }^{12}$ M. Lima, ${ }^{30,18}$ M. A. G. Maia, ${ }^{18,22}$ J. L. Marshall, ${ }^{31}$ P. Melchior, ${ }^{32}$ F. Menanteau, ${ }^{19,20}$ R. Miquel, ${ }^{33,21}$ F. Paz-Chinchón, ${ }^{19,20}$ A. A. Plazas, ${ }^{32}$ E. Sanchez, ${ }^{17}$ V. Scarpine, ${ }^{4}$ S. Serrano,${ }^{24,25}$ I. Sevilla-Noarbe, ${ }^{17}$ M. Smith, ${ }^{34}$ E. Suchyta, ${ }^{35}$ M. E. C. Swanson, ${ }^{20}$ G. Tarle, ${ }^{36}$ V. Vikram, ${ }^{37}$ and J. Weller ${ }^{38,10,11}$

(The DES Collaboration)

${ }^{1}$ Institute for Particle Physics and Astrophysics, ETH Zurich, Wolfgang-Pauli-Strasse 27, CH-8093 Zurich, Switzerland

${ }^{2}$ Department of Astrophysical Sciences, Princeton University, Princeton, New Jersey 08544, USA

${ }^{3}$ Jodrell Bank Center for Astrophysics, School of Physics and Astronomy, University of Manchester, Oxford Road, Manchester M13 9PL, United Kingdom

${ }^{4}$ Fermi National Accelerator Laboratory, P. O. Box 500, Batavia, Illinois 60510, USA

${ }^{5}$ Kavli Institute for Cosmological Physics, University of Chicago, Chicago, Illinois 60637, USA

${ }^{6}$ Department of Physics, Stanford University, 382 Via Pueblo Mall, Stanford, California 94305, USA

${ }^{7}$ Kavli Institute for Particle Astrophysics \& Cosmology, P. O. Box 2450, Stanford University, Stanford, California 94305, USA

${ }^{8}$ National Accelerator Laboratory, Menlo Park, California 94025, USA

${ }^{9}$ Department of Physics \& Astronomy, University College London, Gower Street, London WC1E 6BT, United Kingdom

${ }^{10}$ Max Planck Institute for Extraterrestrial Physics, Giessenbachstrasse, 85748 Garching, Germany

${ }^{11}$ Universitäts-Sternwarte, Fakultät für Physik, Ludwig-Maximilians Universität München, Scheinerstr. 1, 81679 München, Germany

${ }^{12}$ Department of Physics and Astronomy, University of Pennsylvania, Philadelphia, Pennsylvania 19104, USA

${ }^{13}$ Institute for Astronomy, University of Edinburgh, Edinburgh EH9 3HJ, United Kingdom

${ }^{14}$ Instituto de Fisica Teorica UAM/CSIC, Universidad Autonoma de Madrid, 28049 Madrid, Spain

${ }^{15}$ CNRS, UMR 7095, Institut d'Astrophysique de Paris, F-75014 Paris, France

${ }^{16}$ Sorbonne Universités, UPMC Univ Paris 06, UMR 7095, Institut d'Astrophysique de Paris, F-75014 Paris, France

${ }^{17}$ Centro de Investigaciones Energéticas, Medioambientales y Tecnológicas (CIEMAT), Madrid, Spain

${ }^{18}$ Laboratório Interinstitucional de e-Astronomia-LIneA,

Rua Gal. José Cristino 77, Rio de Janeiro, RJ-20921-400, Brazil

${ }^{19}$ Department of Astronomy, University of Illinois at Urbana-Champaign, 1002 W. Green Street, Urbana, Illinois 61801, USA

${ }^{20}$ National Center for Supercomputing Applications, 1205 West Clark Street, Urbana, Illinois 61801, USA

${ }^{21}$ Institut de Física d'Altes Energies (IFAE), The Barcelona Institute of Science and Technology, Campus UAB, 08193 Bellaterra (Barcelona) Spain

${ }^{22}$ Observatório Nacional, Rua Gal. José Cristino 77, Rio de Janeiro, RJ-20921-400, Brazil

${ }^{23}$ Department of Physics, IIT Hyderabad, Kandi, Telangana 502285, India

${ }^{24}$ Institut d'Estudis Espacials de Catalunya (IEEC), 08034 Barcelona, Spain

${ }^{25}$ Institute of Space Sciences (ICE, CSIC), Campus UAB,

Carrer de Can Magrans, s/n, 08193 Barcelona, Spain

${ }^{26}$ Santa Cruz Institute for Particle Physics, Santa Cruz, California 95064, USA

${ }^{27}$ Center for Cosmology and Astro-Particle Physics, The Ohio State University, Columbus, Ohio 43210, USA

${ }^{28}$ Department of Physics, The Ohio State University, Columbus, Ohio 43210, USA

${ }^{29}$ Center for Astrophysics I Harvard \& Smithsonian,

60 Garden Street, Cambridge, Massachusetts 02138, USA

${ }^{30}$ Departamento de Física Matemática, Instituto de Física, Universidade de São Paulo, CP 66318, São Paulo, SP 05314-970, Brazil 


\author{
${ }^{31}$ George P. and Cynthia Woods Mitchell Institute for Fundamental Physics and Astronomy, and \\ Department of Physics and Astronomy, Texas A\&M University, College Station, Texas 77843, USA \\ ${ }^{32}$ Department of Astrophysical Sciences, Princeton University, \\ Peyton Hall, Princeton, New Jersey 08544, USA \\ ${ }^{33}$ Institució Catalana de Recerca i Estudis Avançats, E-08010 Barcelona, Spain \\ ${ }^{34}$ School of Physics and Astronomy, University of Southampton, Southampton SO17 1BJ, United Kingdom \\ ${ }^{35}$ Computer Science and Mathematics Division, Oak Ridge National Laboratory, \\ Oak Ridge, Tennessee 37831, USA \\ ${ }^{36}$ Department of Physics, University of Michigan, Ann Arbor, Michigan 48109, USA \\ ${ }^{37}$ Argonne National Laboratory, 9700 South Cass Avenue, Lemont, Illinois 60439, USA \\ ${ }^{38}$ Excellence Cluster Origins, Boltzmannstr. 2, 85748 Garching, Germany
}

(Received 27 June 2019; accepted 5 April 2020; published 27 April 2020)

\begin{abstract}
Weak lensing by large-scale structure is a powerful probe of cosmology and of the dark universe. This cosmic shear technique relies on the accurate measurement of the shapes and redshifts of background galaxies and requires precise control of systematic errors. Monte Carlo control loops (MCCL) is a forward modeling method designed to tackle this problem. It relies on the ultra fast image generator (UFig) to produce simulated images tuned to match the target data statistically, followed by calibrations and tolerance loops. We present the first end-to-end application of this method, on the Dark Energy Survey (DES) Year 1 wide field imaging data. We simultaneously measure the shear power spectrum $C_{\ell}$ and the redshift distribution $n(z)$ of the background galaxy sample. The method includes maps of the systematic sources, point spread function (PSF), an approximate Bayesian computation (ABC) inference of the simulation model parameters, a shear calibration scheme, and a fast method to estimate the covariance matrix. We find a close statistical agreement between the simulations and the DES Y1 data using an array of diagnostics. In a nontomographic setting, we derive a set of $C_{\ell}$ and $n(z)$ curves that encode the cosmic shear measurement, as well as the systematic uncertainty. Following a blinding scheme, we measure the combination of $\Omega_{m}, \sigma_{8}$, and intrinsic alignment amplitude $A_{\mathrm{IA}}$, defined as $S_{8} D_{\mathrm{IA}}=\sigma_{8}\left(\Omega_{m} / 0.3\right)^{0.5} D_{\mathrm{IA}}$, where $D_{\mathrm{IA}}=1-0.11\left(A_{\mathrm{IA}}-1\right)$. We find $S_{8} D_{\mathrm{IA}}=0.895_{-0.039}^{+0.054}$, where systematics are at the level of roughly $60 \%$ of the statistical errors. We discuss these results in the context of earlier cosmic shear analyses of the DES Y1 data. Our findings indicate that this method and its fast runtime offer good prospects for cosmic shear measurements with future wide-field surveys.
\end{abstract}

DOI: $10.1103 /$ PhysRevD.101.082003

\section{INTRODUCTION}

Recent observations combining different cosmological probes have led to the establishment of the $\Lambda \mathrm{CDM}$ concordance model for cosmology. One of these probes is cosmic shear, the measurement of spatial correlations in the apparent shape of background galaxies due to the weak gravitational lensing effect. Since the first statistical detections of the effect [1-4], there have been a large number of measurements with larger sample sizes and improved accuracies. Recently, several wide-field surveys have reported cosmic shear measurements with unprecedented accuracies, such as the Kilo Degree Survey (KiDS) [5], the Subaru HSC survey [6] and the Dark Energy Survey (DES) [7].

A key requirement in cosmic shear measurements is the control of systematics both for the measurement of the shear correlation function and for the redshift distribution

\footnotetext{
*Corresponding author. tomasz.kacprzak@phys.ethz.ch
}

$n(z)$ of the galaxy sample used for the shape measurements. To tackle this problem, a number of shape measurement methods have been proposed [8-11] and have been reaching an increasing precision. In parallel, various photometric redshift methods have been developed to derive galaxy redshifts from multiband imaging data [12-16].

Recently, the Monte Carlo control loop [17] (MCCL) method was proposed to tackle the shear and $n(z)$ measurement jointly. It is based on a forward modeling approach using the ultra fast image generator (UFig) [18]. In this method, image simulations are first tuned to agree statistically with the target data set and then used to calibrate the cosmic shear measurement and to quantify its systematic uncertainty. The method was first tested at the 1-point [19] and 2-point level [20] using simulations as mock observed data, which were also used to study the propagation of systematic effects onto the final cosmic shear measurement. The MCCL method was also used to determine the redshift distribution of cosmological samples of galaxies [21]. Recently, the galaxy population model 
resulting from the MCCL method derived from broadband imaging data was successfully compared to the Sloan Digital Sky Survey (SDSS) spectroscopic sample [22] and the narrow band imaging Physics of the Accelerating Universe Survey (PAUS) [23].

In this paper, we present the first end-to-end cosmological analysis using the MCCL method, applied to the DES Year 1 (Y1) survey. It constitutes a nontomographic reanalysis of this data set with an independent approach. We start from coadded images and perform object detection, point spread function (PSF) modeling, shear calibration, $n(z)$ measurement, covariance matrix calculation, power spectra measurement and cosmological likelihood analysis. At the heart of this approach is the simultaneous measurement of the shear angular power spectrum $C_{\ell}$ and the redshift distribution $n(z)$ of the galaxy sample. After describing the method and its specific implementation for DES Y1, we present our results and cosmological constraints, and compare our results to the earlier DES Y1 analysis. We follow a blinding scheme throughout our work. Finally, we discuss the application of our method to future data sets, such as DES future releases.

This paper is organized as follows. In Sec. II, we review the main features of the MCCL approach. In Sec. III, we describe how the simulations are matched to the data. Our measurements of the weak lensing power spectrum and the redshift distribution of the lensed galaxies is presented in Sec. IV. We present our cosmology constraints in Sec. V and conclusions in Sec. VII. The appendix describes our blinding scheme, the PSF modeling, the implementation of approximate Bayesian computation (ABC), and the internal tests on simulations.

\section{MONTE CARLO CONTROL LOOPS METHODOLOGY}

In [17], a framework called Monte Carlo control loops was presented as a method for making robust cosmological measurements. The key principle of this approach is to heavily rely on realistic simulations and to analyze simulations in exactly the same way as is done for the observations. In doing this, we are able to rigorously test all aspects of the measurement process in the regime used in the analysis. The MCCL method divides the measurement process into three key steps that we identify as control loops. In the first step, control loop 1, the simulations are tested against the data using a set of diagnostics to ensure that the simulations have a high fidelity to the data in the spanned space. The forward model includes the intrinsic galaxy population and the Milky Way (stars and dust), as well as measurement features, such as the point spread function (PSF) and noise properties of the images. The result of this first step is a set of model configurations that agree with the data. In the second step of the MCCL process, control loop 2, these simulations are used to calibrate the galaxy shear and redshift measurement sections of the pipeline. In the third step, control loops 3.1 and 3.2, the robustness of these measurements is tested by taking excursions away from the fiducial simulation configurations that were used to calibrate the measurements. As well as allowing us to perform a tolerance analysis, this exploration of measurement sensitivities also allows us to account for uncertainty stemming from systematic errors in a probabilistic way. The shear power spectrum and redshift distribution measurements are then used for cosmological inference that accounts for both statistical and systematic errors.

The implementation of MCCL in this work follows these steps in order to obtain the final cosmology constraint:

(i) we build parametric models for simulating co-added DES images including systematic maps, Milky Way and galaxy populations,

(ii) we find a posterior on the model parameters using $\mathrm{ABC}$,

(iii) we run an ensemble of simulations of the full DES area using the points from the $\mathrm{ABC}$ posterior,

(iv) we calculate shear calibration parameters and redshift distribution for each simulation,

(v) we apply the calculated shear calibration parameters to the galaxy catalogs obtained from the DES images to create a family of pairs of $C_{\ell}$ and $n(z)$ corresponding to the $\mathrm{ABC}$ posterior,

(vi) we calculate cosmology constraints for each pair of $C_{\ell}$ and $n(z)$,

(vii) we combine the ensemble of cosmological constraints to create the final constraint that marginalizes over shear calibration and redshift distribution uncertainties.

Step (i) above corresponds to control loop 1, step (iv) to control loop 2, step (ii) to control loop 3.1, while testing the model extensions in Sec. IV C to loop 3.2. This pipeline was accompanied by an array of tests, such as recovery of input $C_{\ell}$ from simulations, the impact of model extensions, and discrepancies in systematic maps. These tests are described in the sections below. We follow a blinding scheme and define a set of conditions to be met before unblinding in Sec. VE.

\section{FIDUCIAL SIMULATION PARAMETERS (LOOP 1)}

As stated earlier, the MCCL approach implemented in this work relies on modeling of all important features that have an impact on the key measurements of shear and redshifts of galaxies. These include the intrinsic properties of the galaxy population over cosmic time, a model of the Milky Way, and observational features linked to the data taking. In this section, we present a brief description of these components along with our measurement and results that lead to our fiducial simulation parameters for the later work. The method to choose the fiducial simulation is described in Sec. III D. 


\section{A. Galaxy population model}

A detailed description of the features of the intrinsic galaxy population model used in this study is given in [21]. In order to render images of galaxies, we need to assign fluxes, light profiles, and positions to each galaxy. We do this by first modeling the galaxy luminosity distributions of different galaxy populations, red and blue, using Schechter functions $\phi$, which can evolve with redshift. By drawing from these functions, we are able to generate a sample of galaxies with redshifts and absolute magnitudes. Next, we draw a rest-frame spectral energy distribution (SED) for each galaxy. We model the SEDs as a linear combination of five template spectra, which are based on the BruzualCharlot stellar evolution synthesis models [24] and which are also used by KCORRECT [25]. The corresponding coefficients are sampled from a Dirichlet distribution, which is motivated empirically by data from the SDSS, as described in [21]. At this step, we again make a distinction between the red and the blue galaxy populations by using two distinct Dirichlet distributions. After each galaxy has been assigned a spectrum, we are able to compute apparent fluxes in arbitrary filter bands, which are used to render the objects on our simulated images. We also include reddening by Galactic dust using the extinction map derived by [26]. The positions of galaxies on the sky are drawn uniformly, without clustering.

After assigning fluxes to our galaxies, we randomly draw a light profile for each object. We use Sérsic profiles [27] parametrized by the Sérsic index $n$ and the half-light radius $r_{50}$ to model the light distributions of our galaxies. To assign half-light radii, we use the model given by [21], i.e., we sample physical half-light radii for our galaxies from a log-normal distribution with a fixed standard deviation and a mean that depends on the absolute magnitudes of the galaxies. We then transform the physical size to an angular size on the sky using the angular diameter distance, calculated using the fiducial cosmological model.

We assign the same Sérsic index $n_{\text {blue }}$ to all galaxies sampled from the blue population and the same Sérsic index $n_{\text {red }}$ to all galaxies sampled from the red population, whereby $n_{\text {blue }} \neq n_{\text {red. }}$. This is motivated by results from the literature, which suggest that blue galaxies are on average well described by a Sérsic index $n=1$ and red galaxies are well matched using $n=4[28,29]$. The value of $n_{\text {blue }}$ is found using the $\mathrm{ABC}$ scheme, while $n_{\text {red }}=4$ is fixed (see Sec. III D).

Finally, each simulated galaxy is assigned an intrinsic ellipticity described by two components $e=\left(e_{1}, e_{2}\right)$. We do it by drawing an ellipticity magnitude $|e|$ from the $p(|e|)$ distribution and rotate it by a random angle. We use a $p(|e|)$ based on the Beta distribution. Our model uses two parameters: $e_{\text {ratio }}$ and $e_{\text {sum }}$, which map to Beta distribution parameters $\alpha, \beta$ in the following way: $\alpha=e_{\text {sum }} e_{\text {ratio }}$ and $\beta=e_{\text {sum }}\left(1-e_{\text {ratio }}\right)$. Variation in $e_{\text {ratio }}$ corresponds to shifting the mode of the distribution between 0 and 1 . Value of $e_{\text {sum }}$ close to zero results in an distribution that is close to uniform, while large $e_{\text {sum }}$ in a narrow spread around the mode. The prior on these parameters is specified in Appendix D 1. The posterior is found using the $\mathrm{ABC}$ scheme, see Fig. 11.

We assume a cosmological model to calculate the angular diameter distances in the calculation of magnitudes and sizes of galaxies. We use the same cosmological parameters as in [21]. The ABC posterior is constrained by magnitudes, sizes, and colors of the galaxies detected in the images, as well as the spectroscopic redshift sample from VVDS. Using a slightly different cosmology parameters would modify angular diameter and luminosity distances, and these changes would be, to first order, compensated by modifying other model parameters, such as the normalization or redshift evolution of the luminosity and size functions. As these parameters are degenerate and the posterior is anchored on the imaging and spectroscopic data, we do not expect the calculated $n(z)$ and shear calibration to change significantly. Therefore, we do not expect this assumption to influence the cosmological constraints measured in this work. It may prove useful to investigate this dependence in more detail for future lensing surveys.

\section{B. Milky Way model}

To generate a catalog of stars for rendering the simulated image, we combine the stars in the Gaia Data Release 2 (DR2) [30], with the Besançon model [31] of the Milky Way [32]. The Gaia objects are placed on the image according to their actual position on the sky, such that we estimate the PSF in the simulations at the same positions as in the data. To generate the faint end of the stellar population, we use the Besançon model, which is based on stellar population synthesis and reaches the magniture of $r<25$. We evaluate the model for all HEALPix [33] pixels of a map with nside $=8$ that overlap with the DES Y1 area. We create Besançon catalogs that cover an area of $5 \mathrm{deg}^{2}$ and subsample these catalogs according to the area covered by the simulated images. This way the variation in density is included in the simulations.

To combine the stars from Gaia with the ones generated by the Besançon model, we map the apparent CFHTMegaCam magnitudes of the Besançon stars to the Gaia $G$-band using the relation provided in [34] (second equation in Sec. V. B and Table 7). For each Gaia object, we then find the closest match from the Besançon stars in terms of the $G$-band apparent magnitude. The matched Besançon stars are subsequently placed at the positions of the corresponding Gaia objects. While not all objects in the Gaia catalog are true stars, at this stage we do not attempt to improve the purity of the sample. This is, however, addressed at the PSF modeling step (See Appendix C). 


\section{Model of the measurement process}

We analyze coadded DES images, as well as simulate coadds with UFig. We do not analyze single exposure images in our method, although we use information about them to create systematic maps of the PSF and noise in the co-adds. Each exposure taken by the dark energy camera [35] comprises of 62 images, each taken by a single chip. Additional 12 chips are used for guiding and focus. However, the Y1 images were constructed mostly from $67 \mathrm{CCDs}$ due to various instrumental issues [36]. In the DES pipeline, each co-add image is created by adding single chip images from multiple exposures. Before the co-addition, the chip images are resampled to the co-add coordinate system using an astrometric solution [37,38]. Therefore, the coadd image properties, such as noise levels or the PSF, can sharply change across the image in places corresponding to boarders of single chip images. To include this effect in simulations, we create a set of Boolean maps for each coadd, which contains information about each exposure's contribution to each pixel in the coadd. We create the Boolean exposure maps for all griz $Y$ bands and use them to create noise level and PSF maps.

Noise level maps contain information about the noise standard deviation for each pixel in the coadd. They are created using the noise level estimate in the headers of single chip images, based on the SKYSIGMA field. This field contains the standard deviation of the sky background noise. A weighted estimate is created for the coadd pixels with multiple single image contributions using the same weighting scheme as used in the coadd production process. An example noise map is shown in Figure 1. To finetune the noise level, the map is then multiplied by a scaling parameter $s_{\mathrm{bkg}}$, which is found using $\mathrm{ABC}$ (see Appendix D). For each simulated image, we draw the Gaussian noise realization from the noise map. To emulate the effects of the coaddition process on the images, we convolve the drawn noise with a specially designed kernel, which is created so that the autocorrelation of the convolved noise image is similar to that expected from the
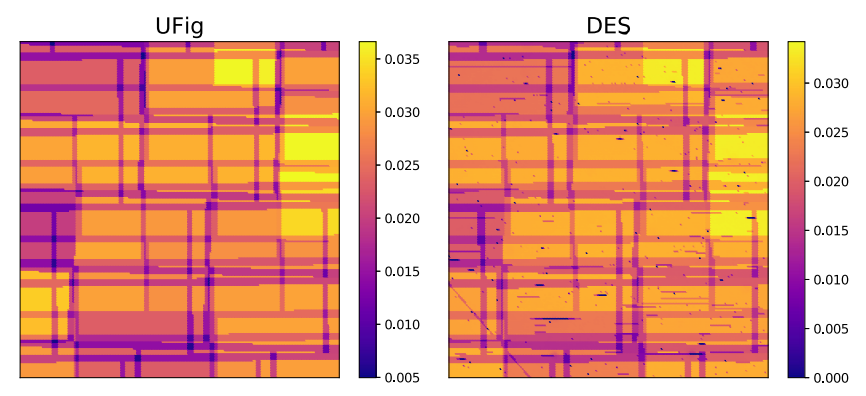

FIG. 1. Example noise map for tile DES0622-6039. The color shows the inverse variance of the pixel noise. The tiling pattern is constructed using the astrometric information of every chip that is used for constructing the co-added image.
Lanczos resampling with $n=3$, as employed by the DES pipeline [37] (see Appendix H for details).

Objects in the images are detected by SEXTRACTOR [39]. We analyze DES and UFig images using the same SEXTRACTOR settings (see Appendix E). While running SEXTRACTOR on DES data, we used the noise maps accompanying the coadd images and produced by the DES pipeline. For simulations, the noise maps are taken from the inverse variance maps described above. We verify that there is no significant difference on measured moments when using one or the other map, as SEXTRACTOR rescales the noise maps internally after performing noise level estimation.

In the DES pipeline, the background is subtracted from each single exposure before coaddition. We simulate the coadds directly according to noise and PSF maps, and do not include a background light model. We do, however, subtract the global mean of the image. To address this slight discrepancy, we turn on both global and local background subtraction in the SEXTRACTOR configuration on the DES images and simulations. We verified that the SEXTRACTOR output is robust to the level of background on the images for our data.

The PSF model is based on three key elements: parametric PSF models, fast parameter measurement with deep learning, and interpolation on coadds. This pipeline is independent of that used in [40], and allows for fast modeling inside the control loops. We measure the PSF parameters only from the objects identified in the Gaia catalogs, with magnitudes in the DES $r$-band $17<m<22$. The PSF model is based on a double Moffat [41] profile, with $\beta_{1}=2$ and $\beta_{2}=5$. It has 9 parameters: size, ellipticity $(2 \mathrm{x})$, flexions $(4 \mathrm{x})$, kurtosis, and the ratio of fluxes between the two Moffat profiles. We obtain the parameters of that model using a deep learning method described in [42], with few modifications (see Appendix C). PSF maps are created using the Boolean exposure maps described above. We interpolate these parameters across the co-add plane using a basis that combines Chebyshev polynomials and the information from the Boolean exposure maps (see Appendix $\mathrm{C}$ for more details). This way, the discontinuities in the PSF variations across the coadd can be included. We use a robust fitting algorithm with a $\sigma$-clipping procedure, which aims to remove unusual stars, including false positives in the Gaia catalog. For each tile, a randomly chosen set of $15 \%$ of the stars is excluded from being used as an input to PSF model fitting. These stars constitute a validation sample, which is used to calculate residuals between the interpolated PSF and measured star parameters. Figure 2 shows an example PSF map for the PSF size and ellipticity parameters. These models are used for making the forward simulations, as well as for the shear measurement.

We simulated the full DES area in the $i$-band using our forward model and used the exact same set of DES and 


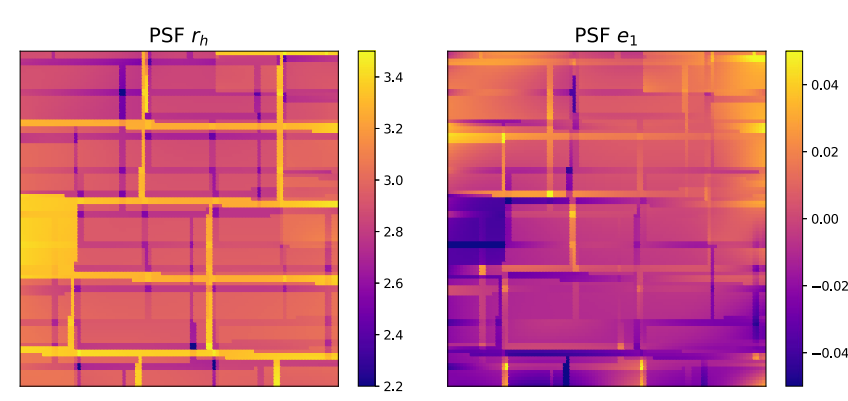

FIG. 2. Example PSF maps for tile DES0622-6039. Maps for PSF FWHM $r_{p}$ and ellipticity $e_{1}$ are shown in the left and right panels, respectively. Maps for flexions, kurtosis and flux ratio from two Moffat profiles are also created in the same way.

UFig tiles to perform our analysis. Figure 3 shows the agreement between simulations and DES data in terms of PSF power spectra. The power spectra are calculated using POLSPICE and described in Appendix B. The parameters of the PSF, calculated at the positions of galaxies, are: PSF FWHM $r_{p}$ and ellipticity $e_{p}$ (top panels). Middle panels show the power spectra of the residual between the PSF estimates, $r_{p}, e_{p}$, and the measurement from validation stars $r_{s}, e_{s}$. Bottom panels show the cross power spectrum between the PSF model and the residual $e_{s}-e_{p}, r_{s}-r_{p}$, at the positions of validation stars. The residual auto and residual $\times$ model power spectra are noise-corrected. The bands correspond to $1 \sigma$ standard deviation and are calculated from multiple realizations of the fiducial survey with different random seeds. The agreement is generally very good for the PSF parameters and the residual power spectra. Small discrepancy is observed in the PSF size residual auto power spectrum. Model $\times$ residual spectra are very low and also in agreement. The PSF power spectra for different simulations vary slightly due to different star sample selection and their measured parameters. This was caused by random selection of validation stars, pixel noise, which affected the star parameter measurement, and blending of the PSF stars with other objects. The differences between these power spectra are, however, very small, and we do not show them here.
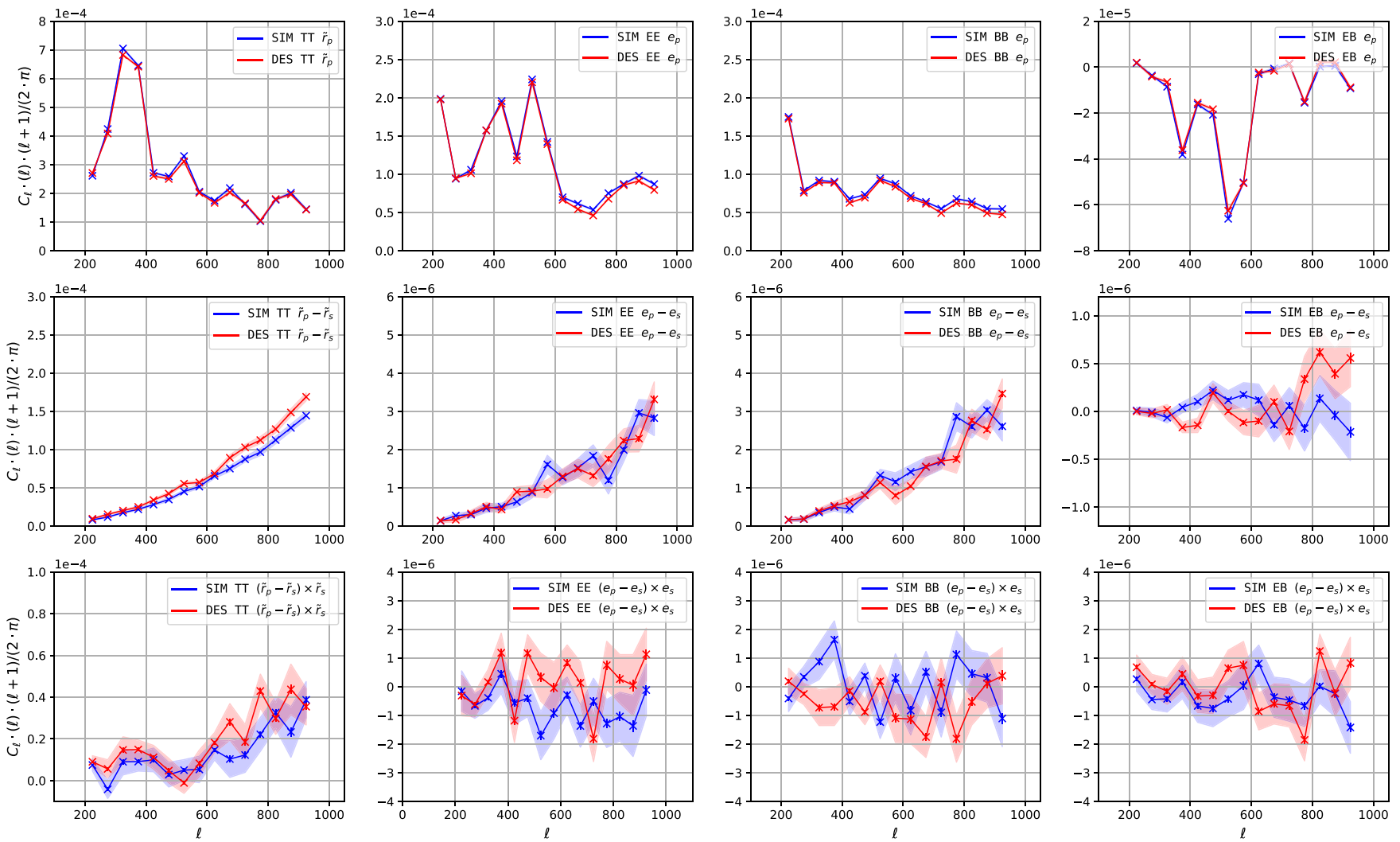

FIG. 3. Agreement between the PSF 2-pt functions in the DES data (red lines) and UFig simulations (blue lines). The upper left panel shows the TT power spectrum for PSF FWHM $\tilde{r}_{p}$, calculated in fractional deviation form $\tilde{r}=(r-\bar{r}) / \bar{r}$, where $\bar{r}$ is the mean size. The remaining upper panels show $\mathrm{EE}, \mathrm{BB}$, and $\mathrm{BE}$ spectra for shape parameters $e_{p}$ of the PSF model calculated at the positions of galaxies. The spectra of the residuals $\tilde{r}_{p}-\tilde{r}_{s}$ and $e_{s}-e_{p}$ between the measured sizes and shapes of validation stars $\tilde{r}_{s}, e_{s}$ and PSF model $\tilde{r}_{p}, e_{p}$ at positions of stars is shown in the middle panels. Cross-spectrum between the residuals $\tilde{r}_{p}-\tilde{r}_{s}, e_{s}-e_{p}$, and PSF estimates $\tilde{r}_{s}, e_{s}$, are shown in the bottom panels. Middle and lower panels show the harmonic space equivalent of $D_{1}$ and $D_{2}$ statistics proposed in [43] as diagnostic tools for PSF model selection. The error-bands correspond to $1 \sigma$ standard deviation calculated from multiple realizations of the fiducial simulation with different random seeds. 


\section{ABC fits to DES data}

We follow the method detailed in [21] to generate a family of image simulations that are statistically consistent with the DES Y1 data. We use approximate Bayesian computation $[44,45]$ to adjust our model to the survey data. It allows for Bayesian inference in situations where the likelihood function is not tractable, which is the case for our simulations: there is no clear empirical expression for the likelihood, neither on the image nor on the catalog level. However, since we are able to compare the simulations to survey data using distance metrics, the $\mathrm{ABC}$ framework allows us to approximate the corresponding true Bayesian posterior.

The parameter space we sample during the $\mathrm{ABC}$ analysis has 35 dimensions. We vary six sets of parameters: (i) the parameters controlling the redshift evolution of the luminosity functions, (ii) the parameters of the Dirichlet distributions used to sample galaxy SEDs, (iii) the parameters of our model for the intrinsic size of galaxies, (iv) the value of the Sérsic index $n_{\text {blue }}$ for galaxies sampled from the blue population, (v) the parameters controlling the distribution from which we sample intrinsic galaxy ellipticities, (vi) a parameter scaling the background level of our simulated images (see Table II for more details). The Sérsic index for red galaxies $n_{\text {red }}$ is fixed because it impacts $n(z)$ and the shear calibration only very weakly. Furthermore, we have little constraining power on this parameter due to difficulty in measuring it with SEXTRACTOR. In Appendix D 1, we give more information on our parameter space and specify the priors.
The distance metrics we use probe basic properties of the simulated images such as number counts, the distribution of measured galaxy magnitudes, sizes and ellipticities as well as galaxy colors. Furthermore, we include spectroscopic data from the VIMOS VLT Deep Survey (VVDS [46-48]) to tighten the constraints on $n(z)$. In total, we use a combination of five distance metrics to obtain a posterior; further information on this is given in Appendix D 2. To compute the distance values for one sample, we evaluate our model on 20 randomly chosen DES tiles in griz filters, which corresponds to an area of $10.7 \mathrm{deg}^{2}$ (we use the same tiles for all samples). We then compute distance metrics tile-by-tile and average the resulting values to reduce the impact of cosmic variance. In total, we evaluate our model for 110000 prior samples.

In Appendix D, we show the $\mathrm{ABC}$ posterior obtained from the analysis described above. We do not show the parameters controlling the coefficient distributions used to assign spectra to galaxies, since we have little constraining power on these parameters, so that we effectively marginalize over them. Furthermore, we compare histograms of various galaxy quantities measured from the DES data and from the posterior simulations in Fig. 4. We find that the DES histograms (red line) lie within the histograms measured from UFig simulations of $\mathrm{ABC}$ posterior (light blue lines). The fiducial simulation is marked with a dark blue line. That is the case for the bulk of the distributions, some small discrepancy is visible in the tails. Small discrepancy is visible in the FLUXERR_AUTO parameter, but the overall shape of the curves match well. The overall
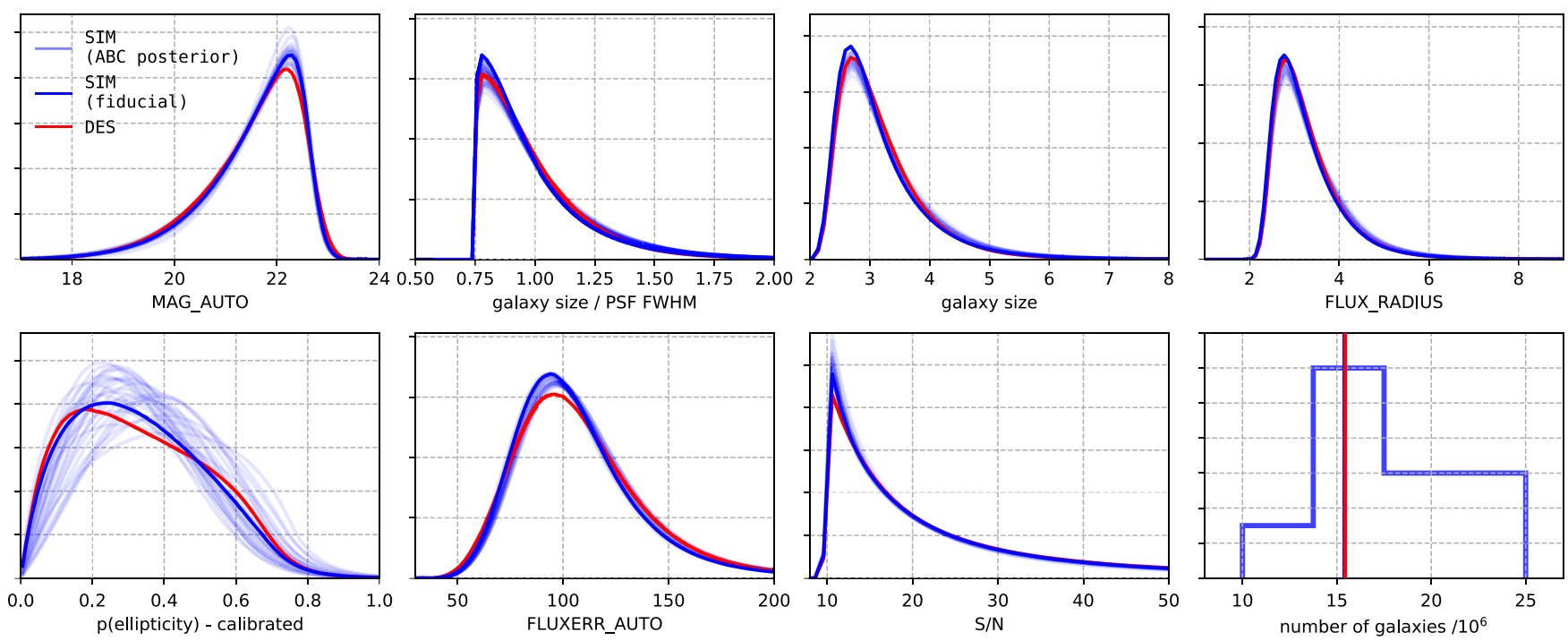

FIG. 4. Agreement between ABC posterior and DES data. The red line shows the histograms from DES data, the light blue lines from the 30 UFig simulations from ABC posterior, and the dark blue line to the fiducial simulated survey. These normalized histograms are created using the full DES catalog, as well as catalogs created from simulating the full area in the $i$-band. The bottom right panel shows the number of galaxies in the DES data (red line) and 30 UFig simulations (blue dots). The y-axis corresponds to the indices of 30 simulations. The galaxy size was calculated using Eq. (E1). The signal-to-noise ratio (S/N) has been calculated from SEXTRACTOR parameters as FLUX_AUTO/FLUXERR_AUTO. 
agreement ensures that the DES data lies within the simulation space, according to our metrics. The uncertainty on the overall number of galaxies and the $p(e)$ is larger than for the other parameters. It can be improved in future work by running the $\mathrm{ABC}$ algorithm for longer until full convergence. However, it is not necessary to decrease this uncertainty further at this point, as the $\mathrm{ABC}$ posterior is always a conservative approximation to the true posterior. Lack of convergence results in a larger systematic uncertainty on $n(z)$ and shear calibration and propagates to the cosmological parameters. The uncertainty in the number of galaxies does not affect the covariance matrix for the DES power spectrum, as it is calculated using the DES shapes directly, as described in Sec. V D.

We choose the sample with the lowest combined distance measure as our fiducial parameter set. This set is then used to create the fiducial simulation, on which many of the following basic tests are performed.

\section{JOINT SHEAR POWER SPECTRUM AND REDSHIFT MEASUREMENT (LOOP 2 AND 3)}

In the MCCL method, we jointly measure the redshift distribution of source galaxies $n(z)$ and the shear power spectrum $C_{\ell}$. To achieve this, we use exactly the same simulated galaxy catalog from UFig simulations to calculate an $n(z)$ distribution and shear calibration parameters. Moreover, by using a set of surveys from the ABC posterior that are compatible with the DES data, we effectively quantify the uncertainty on $n(z)$ and the shear calibration.

Our method is designed to measure the shear power spectrum and $n(z)$ only $[20,21]$. The shear estimates are not designed to be robust to all systematics; in fact, the measurement relies heavily on calibration for a dataset with specific properties. The shear bias as a function of various quantities, like signal-to-noise ratio or galaxy size, may remain nonzero [20]. This way, the calibrated shear catalog can be considered only to be an intermediate product. The confidence about the accuracy of the results stems from the fact that the simulations are well matched to the observations and display similar biases, and that we correctly recover the shear power spectrum in simulations. As long as similar biases are present between the DES and UFig data, and the power spectrum is recovered correctly in the simulations, the DES measurement should be expected to be equally accurate.

We simulate 30 UFig surveys of the full Y1 area using the points from the $\mathrm{ABC}$ posterior, including the fiducial survey. Each of these 30 simulations is used to calculate the redshift distribution and a set of shear calibration parameters. These parameters are then applied to the fiducial UFig catalog and to the DES catalog, and the power spectra are computed. This way, we obtain $30 C_{\ell}$ and $n(z)$ pairs, for both DES and the fiducial UFig survey. Variations in these parameters capture the uncertainty in shear and redshift inside the $\mathrm{ABC}$ posterior.

\section{A. Shear calibration and power spectrum measurement}

We follow the method presented in $[19,20]$ with several modifications. The method in $[19,20]$ uses quantities measured by SEXTRACTOR [39] and PSF parameters to create the shear estimator for each galaxy. In this work, we use the PSF parameters output by the convolutional neural network (CNN), described in [42]. Further modifications include the correction of the effect of the SEXTRACTOR weight function used to measure the quadrupole moments. For the details of SEXTRACTOR run, see Appendix E. Then, we create shear maps using the HEALPix pixelization scheme [33] and measure their power spectrum with POLSPICE [49-51]. This process is described in detail in Appendix B.

\section{Shear measurement and calibration}

We use SEXTRACTOR weighted moments X2WIN_IMAGE, Y2WIN IMAGE and XYWIN IMAGE to create the moment matrix $\overline{\mathbf{M}}$. Similarly, we use the PSF size and ellipticity to create the PSF moment matrix P. To measure the weighted moment, SEXTRACTOR uses a Gaussian weight function with width $\sigma_{w}=0.5 \cdot$ FLUXRADIUS $^{2} / \log (2)$, where FLUX_RADIUS is the measured half-light radius [39]. The estimated, deconvolved galaxy moment is then

$$
\mathbf{Q}=\left[\mathbf{M}^{-1}-\alpha_{2} \mathbf{W}^{-1}\right]^{-1}-\alpha_{1} \mathbf{P},
$$

where $\mathbf{W}$ is diagonal with $W_{i i}=\sigma_{w}$. Parameters $\alpha_{1}, \alpha_{2}$ and $\eta$ (defined below) control the shear calibration and are found using simulations. This equation gives the correct, deconvolved moment for $\alpha_{1}=\alpha_{2}=1$ if the observed galaxy, the PSF, and the weight are all Gaussian. The shear estimators are given by

$$
\begin{aligned}
& \gamma_{1}=\eta \frac{Q_{11}-Q_{22}}{Q_{11}+Q_{22}}, \\
& \gamma_{2}=\eta \frac{2 Q_{12}}{Q_{11}+Q_{22}} .
\end{aligned}
$$

The calibration parameter $\alpha_{2}$ is set to $\alpha_{2}=1$. We vary $\alpha_{1}$ on 200 grid points between $\alpha_{1} \in[0.6,0.8]$ and select the value that minimizes the PSF leakage, for PSF ellipticity binned in 50 bins of equal size between $e_{p}^{\min }=-0.1$ and $e_{p}^{\max }=0.1$. For each value of $\alpha_{1}$ we choose $\eta$ that minimizes the shear multiplicative bias for these bins. For the fiducial simulation, the calibration parameters are: $\eta=0.7616$ and $\alpha_{1}=0.7246$. Across the 30 simulations from the $\mathrm{ABC}$ posterior, we find $\eta=0.7742 \pm 0.0222$ and $\alpha_{1}=0.7280 \pm 0.0050$.

The source galaxy sample is created by applying a range of cuts on galaxy and PSF size ratio, signal-to-noise, SEXTRACTOR flags, maximum ellipticity, and others, as described in Appendix E. The catalogs are created using $i$-band objects only, and contain 15,432,057 objects for 
DES data and 15,370,564 for fiducial UFig survey, and vary slightly when different calibration parameters are applied. This corresponds $\approx 3$ galaxies/ $\operatorname{arcmin}^{2}$ using the definition in [52].

\section{Systematics model}

We assess the quality of the shear measurement by examining the 2-pt statistics: the B-modes and shear-PSF cross power spectra. We additionally investigate shear 1-pt statistics by looking at the mean shear as a function of PSF and galaxy parameters. We find small PSF leakage and significant mean shear for the $\gamma_{1}$ component. We do not expect it to significantly affect the 2-pt measurement, as discussed in Sec. IV D and Appendix G.

We model the systematic contributions to the measured ellipticity $\gamma_{\mathrm{obs}}$ from the PSF in the following way:

$\gamma_{\mathrm{obs}}^{i}=\gamma_{\mathrm{true}}\left(1+\beta_{m}^{i} \delta r_{p}\right)+\alpha^{i} e_{p}^{i} r_{p}^{2}+\beta_{e}^{i} \delta e_{p}^{i} r_{p}^{2}+\beta_{r}^{i} e_{p}^{i} \delta r_{p}^{2}$

where $r_{p}$ is the PSF size, $e_{p}^{i}$ is the PSF shape and $\delta r_{p}, \delta e_{p}^{i}$ are the errors in the PSF model for size and shape, respectively, calculated at the position of validation stars. Coefficient $\alpha^{i}$ quantifies the effect of the error in PSF deconvolution. Coefficients $\beta_{e}, \beta_{r}$ capture the effect of errors in the PSF model. Coefficient $\beta_{m}$ is responsible for multiplicative bias arising from the error in PSF size model. This model is loosely based on the linearized error propagation model in [53] and extends the model used in [54] by adding an additional scaling by PSF size. We can estimate $\alpha$ directly from the data by measuring the slope of $\gamma_{\mathrm{obs}}$ as a function of $e_{p}^{i} r_{p}^{2}$. Coefficients $\beta_{e}, \beta_{r}$ and $\beta_{m}$ can be obtained from simulations, as the true PSF parameters are needed for this measurement. We calculate them by measuring the slope of $\gamma_{\mathrm{obs}}$ as a function of the $\delta e_{p}^{i} r_{p}^{2}$ and $e_{p}^{i} \delta r_{p}^{2}$ for $\beta_{e}^{i}$ and $\beta_{r}^{i}$, respectively. These coefficients should not differ much between the DES data and simulations, as the estimators should respond in the same way to PSF errors for similar galaxy samples. Coefficient $\beta_{m}$ can be obtained from simulations by measuring the slope of multiplicative shear bias as a function of $\delta r$.

We aim for each of these terms to have only a small contribution to the shear $C_{\ell}$, such that the systematic error is smaller than roughly half of the statistical error. For $N_{\ell}=15$ data points chosen in this analysis, this corresponds to a systematic contribution by less than $0.5 / \sqrt{N_{\ell}} \approx 0.15$ of the statistical error to each $C_{\ell}$ vector element; for example $\alpha^{2} C_{\ell}^{e_{e}^{i}} r_{p}^{2}<0.5 \sigma\left[C_{\ell}^{\gamma}\right] / \sqrt{N_{\ell}}$. Note that to achieve a $50 \%$ shift in contours relative to the statistical uncertainty for our $C_{\ell}$ vector, all its elements would have to shift consistently by $15 \%$ in the same direction, if covariance is neglected. We consider this requirement with respect to a measurement that does not include the marginalization of systematic error contributions from shear calibration and $n(z)$ uncertainty. As this marginalization significantly increases the constraints, our requirement can be considered as conservative. Our requirement ignores the cross correlations between the elements of the $C_{\ell}$ vector. As the power spectrum is fairly independent (see Sec. V D) and dominated by the shape noise, we do not expect our requirement calculation to be significantly affected by this simplification.

The cross power spectrum between the measured shear $\gamma_{\mathrm{obs}}$ and each of the additive terms will scale linearly with the coefficient, for example $C_{\ell}^{\gamma \times e_{p}^{i} r_{p}^{2}}=\alpha C_{\ell}^{e_{e}^{i} r_{p}^{2}}$. We use this relation to check the level of systematic contribution against the requirement stated above, by comparing the cross power spectra systematic uncertainty divided by a corresponding coefficient. For example, for the contribution of the deconvolution error, we have

$$
C_{\ell}^{\gamma \times e_{p}^{i} r_{p}^{2}}<\frac{0.5}{\sqrt{N_{\ell}}} \frac{\sigma\left[C_{\ell}^{\gamma}\right]}{\alpha} .
$$

Cross power spectra $\gamma \times \delta e_{p}^{i} r_{p}^{2}$ and $\gamma \times e_{p}^{i} \delta r_{p}^{2}$ should satisfy the same condition, divided by coefficients $\beta_{e}$ and $\beta_{r}$, respectively. It is not possible to estimate the multiplicative contribution from cross power spectra, but we can estimate it directly as $\beta_{m}^{2} C_{\ell}^{\delta r_{p}}$. We calculate the requirement on the multiplicative bias by comparing the diagonal of the covariance matrix with the amplitude of the signal

$$
C_{\ell}^{\delta r_{p}}<\frac{0.5}{\sqrt{N_{\ell}}} \frac{\sigma\left[C_{\ell}^{\gamma}\right]}{C_{\ell}^{\gamma} \beta_{m}^{2}} .
$$

We find $0.15 \sigma\left[C_{\ell}^{\gamma}\right] / C_{\ell}^{\gamma} \approx 0.016$ for the $\ell=200$ using the covariance matrix and the fiducial cosmology power spectrum. Larger $\ell$ have larger requirement. We ignore the contribution of the cross-correlations between the systematics in this calculation, as we found that they are typically very small.

We measure the following values of the coefficients:

$$
\begin{aligned}
\alpha_{\mathrm{DES}}^{1}, \alpha_{\mathrm{DES}}^{2} & =-0.0122,-0.0105 \pm 0.0003 \\
\alpha_{\mathrm{UFig}}^{1}, \alpha_{\mathrm{UFig}}^{2} & =+0.0001,-0.0013 \pm 0.0003 \\
\beta_{e}^{1}, \beta_{e}^{2} & =-0.0646,-0.0673 \pm 0.0007 \\
\beta_{r}^{1}, \beta_{r}^{2} & =-0.109,-0.040 \pm 0.009 \\
\beta_{m}^{1}, \beta_{m}^{2} & =+0.55,+0.39 \pm 0.33 .
\end{aligned}
$$

The leakage parameters $\alpha^{i}$ are calculated from the DES data and the UFig simulations separately. Remaining coefficients are calculated from the simulations. The measurement of $\beta_{m}$ is limited by the number of simulations of the fiducial model, which in our case was 30 . No significant 
asymmetry in these coefficients between the two ellipticity components is found, except for $\beta_{r}^{i}$. The source of this asymmetry may be connected to the asymmetry in mean shear, but is not well understood. We leave it for the future work as both values are within our requirements. In the subsequent calculations we use the higher value out of the two components. For $\beta_{m}$ we use the upper limit $\left|\beta_{m}\right|<1$. The difference between the $\alpha_{\mathrm{DES}}^{i}$ and $\alpha_{\mathrm{UFig}}^{i}$ is not affecting the power spectrum measurement, as both values are below the requirements, as described in the following section.

\section{2-pt statistics}

We calculate the shear power spectrum B-modes, as well as cross-power spectra between shear and PSF ellipticity and size, and residual of these quantities. Figure 5 shows the shear B-mode auto power spectrum (BB) and cross power spectrum between the E- and B-modes (EB). Red and blue lines show the measurement for DES and fiducial UFig survey, respectively, for 30 calibration parameters found from $\mathrm{ABC}$ posterior. The shaded regions correspond to the statistical uncertainty on the measurement. The B-mode of the DES data is found to have reduced $\chi^{2}=0.997$, which corresponds to the $p$-value of $p=0.454$, as calculated using the full B-mode covariance matrix (see Sec. V D). The EB cross correlation is also not statistically significant.

The cross spectra of shear E-mode and PSF parameters are shown in Fig. 6. Again, red and blue lines correspond to the DES and UFig data, respectively. Blue shaded regions signify the $1 \sigma$ uncertainty, measured from 30 random seed realizations of the fiducial UFig survey (see Appendix F for details). The gray lines correspond to the requirement described in Sec. IV A 2, calculated using Eq. (5) and $\alpha_{\mathrm{DES}}^{i}$.
Note that in order to achieve a $0.5 \sigma$ shift in the contours, all the points would have to consistently exceed the requirement in the same direction. This requirement considers only the statistical error and does not include the systematics, which makes it conservative.

There is a slight correlation of the shear with PSF ellipticity $e_{p} r_{p}^{2}$ E-mode, with $\chi_{\text {red }}^{2}=1.79$. This is consistent with the PSF leakage calculated at the 1-pt level. We also notice a significant cross power spectrum between the shear E-mode and PSF size residual B-mode, but that seems to be dominated by one outlier point at $\ell \sim 900$. This trend is not visible for equivalent $\mathrm{EE}$ cross power spectrum. The reduced $\chi^{2}$ for other cross power spectra are generally close to $\chi_{\text {red }}^{2}=1$. It is important to note that the distribution of the cross power spectra is not expected to be Gaussian, and therefore the reduced $\chi^{2}$ may not be the best way to quantify the systematic significance for this problem. We leave this investigation to future work. Nevertheless, none of these cross correlations consistently exceed the given requirements calculated with the coefficients calculated in Sec, IVA 2 in our considered $\ell$ range. This suggests that the PSF is removed well enough for our requirements. The multiplicative error resulting from the error in the PSF size measurement will depend on the coefficient $\beta_{m}$, calculated in Sec, IVA 2 and the PSF size error power spectrum, calculated in Sec, IIIC, so that $C_{\ell}^{\gamma, \text { obs }}=$ $C_{\ell}^{\gamma \text {,true }}\left(1+\beta_{m}^{2} C_{\ell}^{\delta r_{p}}\right)$. We measured $C_{\ell}^{\delta r_{p}}<2 \times 10^{-4}$ and $\left|\beta_{m}\right|<1$, and therefore we expect the multiplicative error stemming from the PSF size error to be smaller than the requirement.

These requirements, however, are exceeded for $\ell$ outside considered range. For low $\ell$, the leakage contribution started to exceed the requirement. For high $\ell$, the PSF ellipticity residual increased greatly which lead to
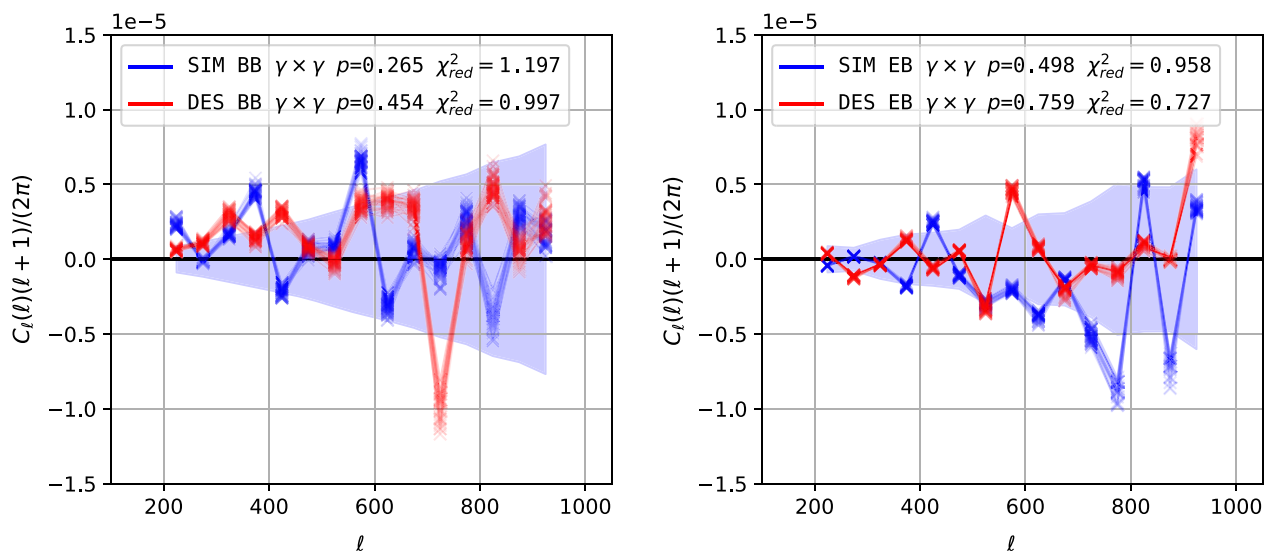

FIG. 5. The shear B-mode auto power spectrum (left panel) and BE cross power (left panel). The red lines correspond to the DES shear catalogs calibrated with 30 different calibration factors from the ABC posterior. The blue lines correspond to fiducial UFig simulation, also calibrated with these 30 factors. The spread between these data points corresponds to the systematic uncertainty. The shaded region corresponds to $1 \sigma$ statistical uncertainty on the measured quantity. For the BB spectrum, these values are taken as the square root of the diagonal of the covariance matrix (see Sec. V D). The uncertainty on the EB spectrum is measured from 30 simulations of the fiducial survey, with different random seeds. 

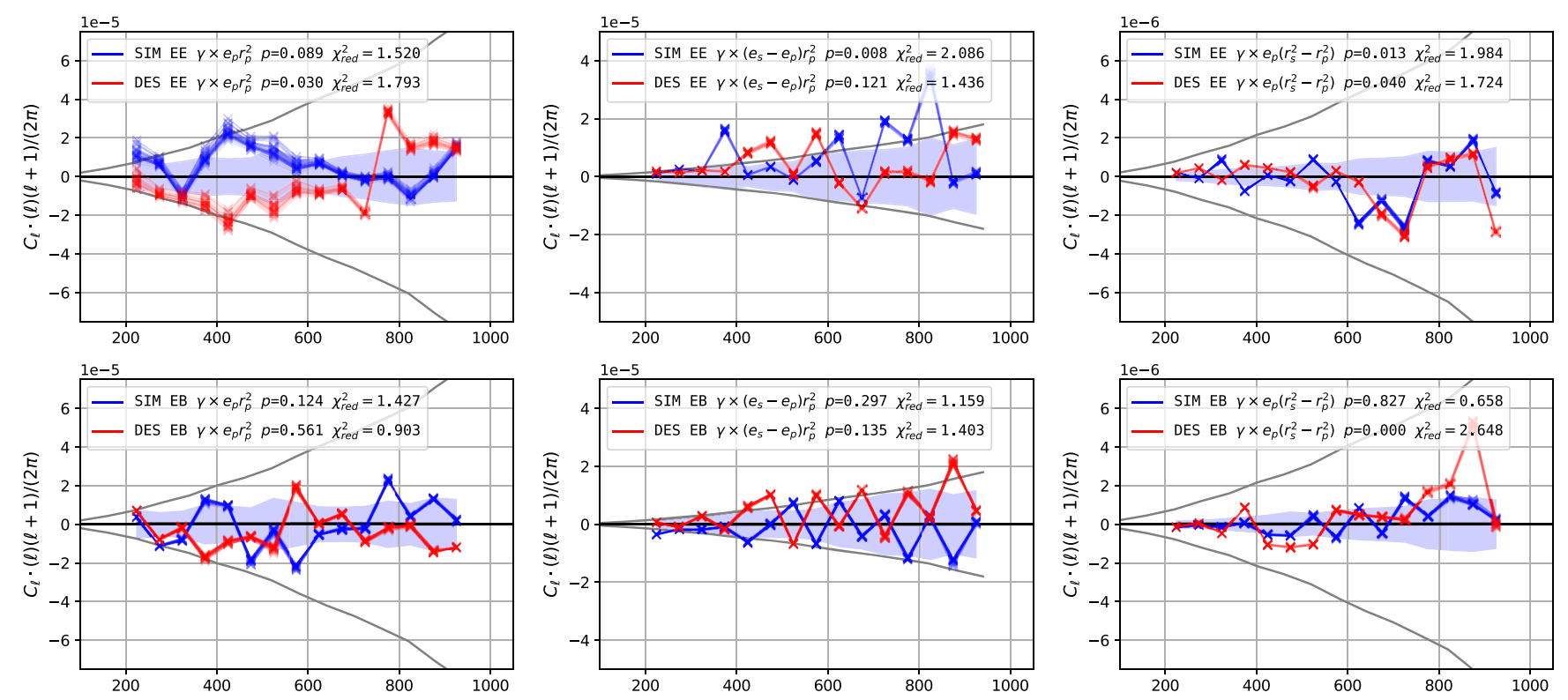

FIG. 6. Diagnostic 2-pt functions of the DES data. The panels show the cross power spectrum between the shear E-mode and PSF model parameters: ellipticity $e_{p}$ and FWHM $r_{p}$ at the galaxy position, as well as the residual between the PSF model and star measurement $e_{s}-e_{p}$ and $r_{s}^{2}-r_{p}^{2}$. Red and blue lines correspond to DES and fiducial UFig simulation, respectively, calibrated with 30 calibration parameter sets from the ABC posterior. The blue error bands correspond to the statistical uncertainty $\sigma_{\text {sys }}^{2}$ and is calculated from the standard deviation of $C_{\ell}$ calculated from 30 simulations of the fiducial survey with different random seeds. The $\chi^{2}$ neglects the covariance between the elements of the $C_{\ell}$ vector. The number of degrees of freedom is $N_{\mathrm{dof}}=15$. The gray lines correspond to the requirements described in Sec. IVA 2. In order to shift the cosmology contours by $0.5 \sigma$, all $C_{\ell}$ elements would have to consistently exceed the requirement. The requirements are conservative, as they are based on statistical uncertainty only, and not including systematic errors from shear calibration and $n(z)$.

$\gamma \times\left(e_{s}-e_{p}\right) r_{p}^{2}$ exceeding the requirements. We therefore decided to limit the $\ell$ range in our analysis to $\ell \in[200,950]$.

The EE power spectrum measured from the DES source galaxy sample is shown on the left panel in Fig. 7. The black line corresponds to the fiducial survey found by the ABC scheme (see Sec. III D). The error bars show the standard deviation estimate, calculated by taking the square root of the diagonal of the covariance matrix. The full covariance matrix of $C_{\ell}$ is shown in Sec. V D. The red lines correspond to the $C_{\ell}$ calculated from the DES sample, but calibrated using 30 different parameter sets, which are obtained from different $\mathrm{ABC}$ posterior points. The size of the source galaxy catalog is varying slightly within the
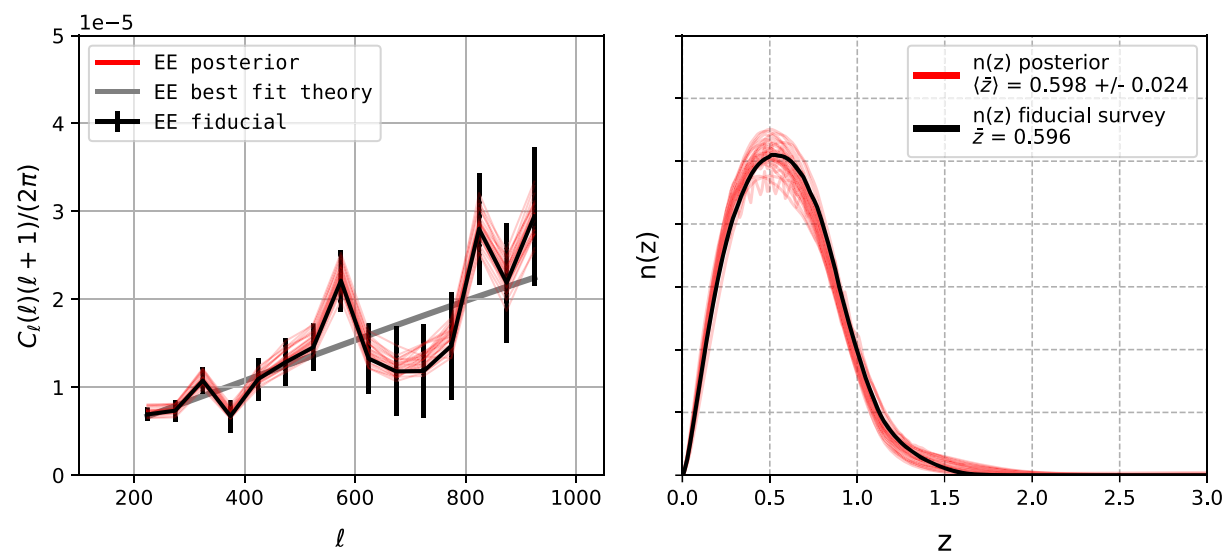

FIG. 7. Joint $\mathrm{n}(\mathrm{z})$ and shear $C_{\ell}$ measured from the DES Y1 data set. Multiple red lines correspond to points from the ABC posterior. The black line shows the measurement using the calibration parameters calculated from the fiducial survey. The error-bars correspond to $1 \sigma$ errors and are taken from the diagonal of the covariance matrix (see Sec. V D). The best fit theory model for the fiducial survey is shown with the gray line. 
30 sets, as each time we removed galaxies with large ellipticities $|e|>1$. The number of galaxies removed this way is typically $<1 \%$. This cut can, in theory, introduce a selection bias, which will be removed by the shear calibration, similarly to other cuts on the galaxy size and $\mathrm{S} / \mathrm{N}$ ratio.

The spread between the $30 C_{\ell}$ measurements represent the systematic uncertainty in our calibration scheme. The $C_{\ell}$ corresponding to the best fit cosmological model for the fiducial survey is shown in gray line. The reduced $\chi^{2}$ of this fit is $\chi^{2}=0.813$.

\section{B. Redshift measurement}

In this analysis we use a single redshift bin, without tomography. We apply the method given in [21] to infer the redshift distribution of our lensing sample. The key idea of this method is to make realistic simulations of the observed data and apply exactly the same image processing pipeline to the observed and the simulated data. This way the detection method and the cuts are the same, which allows us to calculate the $n(z)$ distribution by simply taking the true redshift of all simulated galaxies in the sample. The $\mathrm{ABC}$ uses distance measures based on properties of galaxies in the image data, as well as the distribution of redshifts in the VVDS spectroscopic sample, to obtain the posterior distribution on the input parameters, and consequently corresponding $n(z)$. The VVDS sample is complete to the $i$-band magnitude of $m_{i}<22$. The distance metric that used the VVDS spectroscopic sample compared $i$-band magnitudes and redshifts of UFig galaxies with $m_{i}<22$ in the input catalog, before rendering the images, with the VVDS sample. This was possible due to small errors on the VVDS measurements of redshifts and magnitudes and the completeness of the sample.

Note that this method can only be use to calculate the $n(z)$ distribution of a sample of galaxies, not redshifts of individual galaxies.

In this work, we obtain a posterior set of likely $n(z)$ curves for our lensing sample from the $\mathrm{ABC}$ posterior described in Sec. III D. The redshifts of the galaxies in the simulated lensing samples are then used to construct a family of $30 n(z)$ curves. This result is shown in the right panel of Fig. 7. We compute an average $\langle\bar{z}\rangle$ of mean redshifts $\bar{z}_{i}$ of each curve. Using that ensemble we compute the $\langle\bar{z}\rangle=0.598 \pm 0.024$. The mean $n(z)$ for the fiducial survey is $\bar{z}=0.596$.

A tomographic approach could be straightforwardly accommodated in the MCCL framework. It would nevertheless require more development of the pipeline, which we leave for future work on the following DES data releases.

\section{Robustness to model extensions (loop 3.2)}

The robustness of the measurement can be tested against extensions to the forward model (loop 3.2). If the measurement does not significantly respond to the extension, there is no need to incorporate it into the model. Here we perform one such extension in this work for the fiducial simulation. We allow scatter in the Sérsic index of blue and red galaxies. The Sérsic index is drawn from a truncated normal distribution in the limits between 0.5 and 5 . The mean, before truncation, is set to the original value obtained with $\mathrm{ABC}$, and the standard deviation is set to $\sigma_{n}=0.5$. We run a new simulation with this model using the fiducial parameter set and calculated calibration parameters and $n(z)$ for the extended model the same way as before. We obtain $\alpha_{1}=0.731, \eta=0.783, \bar{z}=0.594$. These parameters are close to the ones calculated from the base model and within the uncertainty calculated from 30 surveys. This indicates that the measurement is robust to this extension and we do not incorporate it into the base model.

\section{Discussion}

The mean shear for the DES and UFig catalogue calculated with fiducial calibration factor is

$$
\begin{aligned}
\left\langle\gamma_{1}^{\mathrm{DES}}\right\rangle,\left\langle\gamma_{2}^{\mathrm{DES}}\right\rangle & =-0.00203,0.00077 \pm 0.00007 \\
\left\langle\gamma_{1}^{\mathrm{SIM}}\right\rangle,\left\langle\gamma_{2}^{\mathrm{SIM}}\right\rangle & =-0.00049,0.00053 \pm 0.00007 .
\end{aligned}
$$

The dispersion in the mean shear from cosmic variance is expected on the level of $\sigma[\langle\gamma\rangle] \approx 2 \times 10^{-4}$. The mean shear in DES catalog is significantly higher than expected from the cosmic variance. For simulations, the mean shear is consistent with cosmic variance. The mean shear, however, does not influence the power spectrum analysis, as we found that it affects only $\ell<100$, and is subtracted before calculating the power spectrum. More details about 1-pt statistics can be found in Appendix G. Nevertheless, it can be a sign of remaining systematics. We look for the source of this mean shear by examining its dependence of various effects that can cause systematic ellipticity shifts. We consider 17 possible variables, including brightness, colors, PSF parameters, distance to bright stars, and position in the footprint. We do not find any significant trends that are not present in the simulations. Given that the mean shear seems to be independent of the systematic variables, we conclude that it should not have a significant impact on the 2-pt measurements.

The difference in PSF leakage $\alpha^{i}$ measured from the DES data and the simulations, described in Sec. IV A 2, may be a sign of a small imperfections in the forward model used in this work. Therefore, it can be the case that the parameters $\beta_{e}, \beta_{r}$, and $\beta_{m}$, which are measured from simulations, are slightly inaccurate for the survey data. However, we do not expect the $C_{\ell}^{\gamma}$ measurement to be affected by that: the crosscorrelations with PSF size and shape error are generally consistent with zero, and the multiplicative bias stemming from PSF size error $\delta r_{p}$ is well within the requirement. 
The discrepancy in $\beta_{m}$ would have to be of order 10 for the requirement to be exceeded.

\section{COSMOLOGICAL CONSTRAINTS}

We measure the cosmological parameters from the nontomographic shear power spectra. We focus on the flat $\Lambda \mathrm{CDM}$ model and vary 5 cosmological parameters $\boldsymbol{\theta}=\left\{h, \Omega_{\mathrm{m}}, \Omega_{\mathrm{b}}, n_{\mathrm{s}}, \sigma_{8}\right\}$, where $h$ is the dimensionless Hubble parameter, $\Omega_{\mathrm{m}}$ is the fractional matter density today, $\Omega_{\mathrm{b}}$ is the fractional baryon density today, $n_{\mathrm{s}}$ denotes the scalar spectral index and $\sigma_{8}$ is the r.m.s. of linear matter fluctuations in spheres of comoving radius $8 h^{-1} \mathrm{Mpc}$.

\section{A. Theory prediction with PyCosmo}

To compute theoretical predictions for cosmic shear power spectra, we follow [55,56] and use the Limber approximation [57-59], which is a valid approximation for large multipoles, typically $\ell>\mathcal{O}(10)$, and broad redshift bins [60]. The expression for the spherical harmonic power spectrum $C_{\ell}^{\gamma \gamma}$ at angular multipole $\ell$ can be found in [55]. We compute all theoretical predictions using PyCosmo [61] and use the transfer function derived by [62] to calculate the linear matter power spectrum. To compute the non-linear matter power spectrum we use the HMCODE fitting function $[63,64]$. To account for the effects of the survey mask, we multiply the predicted $C_{\ell}$ with the POLSPICE kernels, which are output during the $C_{\ell}$ calculation from the survey data (POLSPICE command argument kernelsfileout). The power spectrum is then binned into 15 linearly spaced bins, from $\ell_{\min }=200$ to $\ell_{\max }=950$. The choice of scales is informed by requirements on systematic errors, described in Sec. IVA 2.

\section{B. Intrinsic alignments}

To model the intrinsic alignments, we implement the "nonlinear linear alignment model" [65], which is used in $[54,66,67]$. It consists of two contributions, from intrinsicintrinsic (II) and shear-intrinsic $(G I)$ shape correlations, so that the measured $C_{\ell}^{\text {obs }}$ is

$$
C_{\ell}^{\mathrm{obs}}=C_{\ell}^{\gamma \gamma}+A_{\mathrm{IA}}^{2} C_{\ell}^{I I}+A_{\mathrm{IA}} C_{\ell}^{G I},
$$

where $A_{\mathrm{IA}}$ is the intrinsic alignment amplitude parameter. The impact of $A_{\mathrm{IA}}$ is almost perfectly degenerate with the $\sigma_{8}^{2}$ for a non-tomographic measurement in the $C_{\ell}$ range considered. That is why, in this work, we put constraints on a combination of $\sigma_{8}$ and $A_{\mathrm{IA}}$, and report the product $\sigma_{8} D_{\mathrm{IA}}$, where $D_{\mathrm{IA}}$ is a scaling factor dependent on the strength of intrinsic alignment $A_{\mathrm{IA}}$, as described below.

To do this, we first calculate the ratio between the intrinsic alignment and shear power spectra for our fiducial cosmological model parameters and $A_{\mathrm{IA}}=1$. We find that it is a constant fraction to a good approximation: $C_{\ell}^{I I}=f_{1} C_{\ell}^{\gamma \gamma}$ and $C_{\ell}^{G I}=f_{2} C_{\ell}^{\gamma \gamma}$, where $f_{1}=0.019$ and $f_{2}=-0.117$. As $\sigma_{8}^{2}$ is proportional to $C_{\ell}^{\gamma \gamma}, \sigma_{8}$ will depend on deviation from $A_{\mathrm{IA}}=1$ in the following way

$$
\sigma_{8}\left(A_{\mathrm{IA}}=1\right) \approx \sigma_{8}\left(A_{\mathrm{IA}}\right)\left[\frac{1+f_{1} A_{\mathrm{IA}}^{2}+f_{2} A_{\mathrm{IA}}}{1+f_{1}+f_{2}}\right]^{1 / 2}
$$

and at the fiducial cosmology can be further linearized to:

$$
\sigma_{8}\left(A_{\mathrm{IA}}=1\right) \approx \sigma_{8}\left(A_{\mathrm{IA}}\right) \cdot D_{\mathrm{IA}}
$$

where $D_{\mathrm{IA}}=1-0.11\left(A_{\mathrm{IA}}-1\right)$ is the parameter that scales $\sigma_{8}$ according to the intrinsic alignment amplitude.

\section{Baryonic corrections}

For the baryonic corrections we use prescription in [63], who parametrizes the effects of baryons on the nonlinear power spectrum. Specifically, we follow the implementation of [54], who uses a flat prior in the range $B_{\text {baryon }} \in$ $[2,4]$ of [63]. This range corresponds to feasible range associated with different baryonic feedback models, as seen with hydrodynamic simulations. The dark matter only case corresponds to $B_{\text {baryon }}=3.13$. The second baryon correction parameter $\eta_{0}$ in [63] is set by the equation 30 in that paper.

\section{Covariance matrix estimation with L-Picola}

We compute the covariance matrix used for the likelihood analysis by following the method described in [68]. The method used here avoids the survey geometry-related effects described in [69]. The matter density field is simulated using the fast approximate $N$-Body code L-Picola [70] with the number of particles $N_{\text {part }}=1024$ and the mesh density set to $N_{\text {mesh }}=2048$ per side of the simulation volume. The all-sky past-lightcone is constructed by fixing the observer at the center of the simulation volume and by slicing the volume between $z=0.0$ and the final redshift of $z=1.5$ with no gaps into comoving concentric spherical shells of thickness $\Delta \chi_{b}=$ $\chi\left(z_{b}+\Delta z\right)-\chi\left(z_{b}\right)$ with a redshift-shell thickness of $\Delta z=0.01$, whereas $z_{b}$ is the redshift of the particles within the shell of index $b$. We do not replicate the simulation volume for this construction, since this could potentially lead to statistical artifacts in the power spectra and therefore in the covariance matrix. Furthermore, in this way we ensure that super-survey modes are correctly captured. In order to obtain accurate results for the spherical harmonic power spectra up to angular scales of $\ell \sim 10^{3}$, we nest three simulation volumes with different box-sizes $L_{1}=700 \mathrm{Mpc} / h, L_{2}=4.2 \mathrm{Gpc} / h$ and $L_{3}=$ $6.3 \mathrm{Gpc} / h$. This is done by first constructing the lightcone in the smallest simulation volume from redshift 0.0 to its edge at 0.1 and then continuing the construction in the larger volume from 0.1 to 0.8 using $L_{2}$ and from 0.8 to 1.5 using $L_{3}$. 
We run 10 L-Picola realizations with different seeds (running three simulations with box-sizes $L_{1}, L_{2}$ and $L_{3}$ per realization) and applied the above described pipeline to calculate 10 full-sky convergence maps using the $n(z)$ distribution obtained using the method described in Sec. IV B. Each map is processed with the DES Y1 survey mask resulting in 10 nonoverlapping patches on the sphere. We then add 10 different shape noise realizations to each patch and compute the cosmic shear power spectra. The shape noise is created by rotating the galaxy ellipticity by a random angle, similarly to the noise-correction to the power spectrum (see Appendix B). The covariance matrix used for the analysis it then computed using 1000 realizations (10 maps $\times 10$ patches $\times 10$ noise realizations) of the cosmic shear power spectra for the same binning as for the measurement. The resulting correlation matrix for the shear power spectrum is shown in Fig. 8. The left panel shows the $C_{\ell}$ correlation matrix when no shape noise is added, while the right panel includes the shape noise, which is used in the likelihood. We notice that the covariance matrix is dominated by the shape noise contribution.

\section{E. Blinding}

Throughout the analysis we follow the blinding scheme applied to the EE shear power spectrum, described in Appendix A. We define the following set of quality conditions to be met by our measurement before unblinding: (Q1) properties of the DES galaxy population have to lie within the space covered by the simulations, (Q2) for the fiducial simulation, the input cosmology should be accurately recovered, (Q3) the impact of the possible discrepancies in systematic maps can not be larger than the statistical errors on the measurement, (Q4) small shear B-mode and small cross power spectra between shear and PSF, and (Q5) analysis versions that include well-motivated extensions to the model should not cause significant difference in final result. We review the satisfaction of these conditions in Appendix A and conclude that these

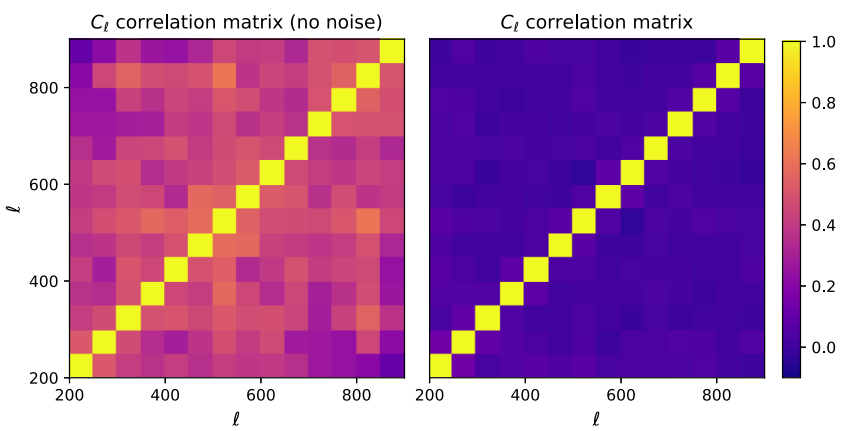

FIG. 8. Correlation coefficient matrix of the power spectrum for angular scales $10^{2}<\ell<10^{3}$ using the $n(z)$ for the fiducial survey (see Sec. IV B) and a binning of $\Delta \ell=50$ using 1000 realizations including shape noise in the right plot and using 100 realizations without shape noise in the left plot. conditions are met. Additional actions taken after the unblinding are documented (Appendix A).

\section{F. Constraints}

We obtain the posterior distribution on cosmological parameters for each of the $30 n(z)$ and $C_{\ell}$ pairs. We create the final constraint with $n(z)$ and shear calibration uncertainty marginalized by adding together the 30 posterior probabilities.

To compute cosmological parameter constraints, we assume the cosmic shear likelihood to be Gaussian, with log-likelihood of the following form

$\mathcal{L}(D \mid \theta)=\frac{1}{|2 \pi \Sigma|^{\frac{1}{2}}}-\frac{1}{2}\left(C_{\ell}^{\mathrm{obs}}-C_{\ell}^{\mathrm{the}}\right)^{\mathrm{T}} \Sigma^{-1}\left(C_{\ell}^{\mathrm{obs}}-C_{\ell}^{\mathrm{the}}\right)$

where $C_{\ell}^{\mathrm{obs}}$ and $C_{\ell}^{\text {the }}$ are the power spectra from observations and theory, respectively, $\Sigma$ denotes the covariance matrix.

We assume flat priors on the cosmological parameters: $h \in[0.5,1.0], \quad \Omega_{m} \in[0.2,0.5], \quad \Omega_{b} \in[0.01,0.09], \quad n \in$ $[0.75,1.25], \sigma_{8} \in[0.5,1.1], B_{\text {baryon }} \in[2.0,4.0]$. The intrinsic alignment parameter was fixed to $A_{\mathrm{IA}}=1$ for the main result, or sampled inside the uniform prior $A_{\mathrm{IA}} \in[-5,5]$.

To obtain the posterior distribution, we evaluate 200,000 likelihoods in 6D parameter space at points chosen from a Sobol sequence [71]. A Sobol sequence is a set of points in a high-dimensional space designed such that the projections of the space are sampled as regularly as possible. Sobol sequence integration is an effective technique to approximate multidimensional integrals [72]. The Sobol sequence was continued for each of the $30 C_{\ell}$ and $n(z)$ pairs, such that likelihoods for each pair are evaluated at different points in this space. We use this technique instead of Monte Carlo Markov Chain (MCMC), as the total turnaround time of this calculation, including queuing time, with single-core jobs is faster and more stable than the parallelized MCMC, despite larger number of likelihood evaluations. To create a 2D posterior for a cosmological parameter pair, we calculate a 2D histogram of all the Sobol sequence points, weighted by their probability. That histogram is then normalized and the confidence intervals are calculated. The posteriors from 30 surveys capture the uncertainty from $n(z)$ and shear calibration, and to marginalize this uncertainty we add the 30 PDFs. This corresponds to discretizing the integral

$$
p\left(\theta_{1}\right)=\int d \theta_{2} p\left(\theta_{1} \mid \theta_{2}\right) p\left(\theta_{2}\right) \approx \sum_{\theta_{2}^{i} \sim p\left(\theta_{2}\right)} p\left(\theta_{1} \mid \theta_{2}^{i}\right)
$$

where $\theta_{1}$ are the parameters of interest and $\theta_{2}$ are the nuisance parameters, and $\theta_{2}^{i} \sim p\left(\theta_{2}\right)$ is a set of samples from $p\left(\theta_{2}\right)$. Note that individual distributions $p\left(\theta_{1} \mid \theta_{2}^{i}\right)$ are not normalized. 

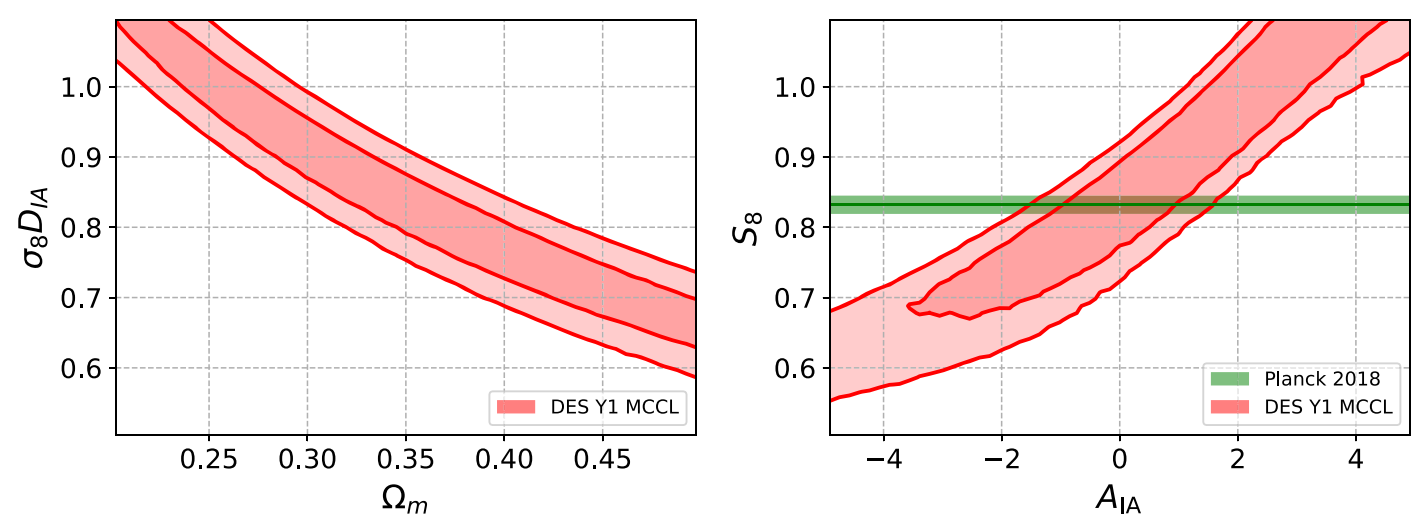

FIG. 9. Cosmological constraints on $\Omega_{m}$ and $\sigma_{8} D_{\text {IA }}$ marginalized over $n(z)$ and shear uncertainties (left panel). The parameter $D_{\text {IA }}=1-0.11\left(A_{\text {IA }}-1\right)$ depends on the amplitude of intrinsic alignments (see Sec. V B). The lines show the $68 \%$ and $95 \%$ confidence intervals. The right panel shows the $S_{8}$ parameter constraint as a function of the intrinsic alignment amplitude. The green bar corresponds to the Planck 2018 measurement of $S_{8}$ (TT,TE,EE + lowE + lensing) [73].

In this nontomographic, cosmic shear only analysis we can only effectively constrain the combination of $\Omega_{m}, \sigma_{8}$, and $A_{\mathrm{IA}}$. Other parameters remain unconstrained. The left panel on Fig. 9 shows the constraints in the $\Omega_{m}-\sigma_{8} D_{\text {IA }}$ plane with marginalized uncertainty on $n(z)$ and shear calibration. The lines represent $68 \%$ and 95\% confidence intervals. The shape of the contour follows a degeneracy characteristic for $\Omega_{m}-\sigma_{8}$. We calculate the constraint on the combination of these parameters $S_{8} D_{\mathrm{IA}}=$ $\sigma_{8}\left(\Omega_{m} / 0.3\right)^{0.5} D_{\text {IA }}$. For this fiducial configuration, we find $S_{8} D_{\text {IA }}=0.895_{-0.039}^{+0.054}$. The right panel on Fig. 9 shows the dependence of the $S_{8}$ constraint on the intrinsic alignment amplitude $A_{\mathrm{IA}}$. There is a clear degeneracy between those parameters. The constraint from the Planck survey [73] is shown with the green bar (TT,TE,EE + lowE + lensing).

We calculate the results for other analysis variants and summarize them in Table I. When the systematic uncertainty is ignored, we find $S_{8} D_{\mathrm{IA}}=0.881_{-0.033}^{+0.050}$ for the fiducial survey. This indicates that the contribution of the systematic uncertainty to the error budget is on the level of $60 \%$ of the statistical uncertainty. The main source of systematic uncertainty is the shear calibration, but it does not dominate it completely. We calculated the standard deviation $\sigma\left[C_{\ell}^{\text {the }}\right]$ of theory power spectrum predicted from the same cosmological parameter set using $30 n(z)$, and the standard deviation of the measurement $\sigma\left[C_{\ell}^{\mathrm{obs}}\right]$ when 30 calibration parameters are applied. We find that $\sigma\left[C_{\ell}^{\text {the }}\right] / \sigma\left[C_{\ell}^{\text {obs }}\right] \approx 0.6$ for large scales, and decreases to $\approx 0.05$ for small scales.

We give the main result for the $\alpha=0.5$ in the $S_{8}=$ $\sigma_{8}\left(\Omega_{m} / 0.3\right)^{\alpha}$ definition in order to easily compare with other measurements. We find, however, that the best-fitting value of the $\alpha$-parameter is $\alpha=0.6$ and gives slightly narrower constraint, $S_{8} D_{\mathrm{IA}}=\sigma_{8}\left(\Omega_{m} / 0.3\right)^{0.6}$ $D_{\text {IA }}=0.907_{-0.040}^{+0.047}$.

\section{G. Comparison with previous DES measurements}

The width of the contours is larger in our analysis than in main DES Y1 [74], mainly due to different choices of the

TABLE I. Comparison of $S_{8} D_{\text {IA }}$ measurements from the non-tomographic MCCL analysis with the tomographic DES Y1 cosmic shear [74], which is additionally marginalized over the uncertainty in the intrinsic alignment modeling. Parameter $D_{\mathrm{IA}}=$ $1-0.11\left(A_{\mathrm{IA}}-1\right)$ controls the strengths of intrinsic alignment, so that $D_{\mathrm{IA}}=1$ for $A_{\mathrm{IA}}=1$. The $\alpha$ parameter in the $S_{8}$ definition was set to $\alpha=0.5$, similarly to the previous DES Y1 analysis [74], unless otherwise stated. We find the best-fitting $\alpha$ parameter in the $S_{8}$ definition to be $\alpha=0.6$. See Sec. V G for details about the $C_{\ell}$ analysis with IM3SHAPE.

\begin{tabular}{lc}
\hline \hline Analysis variant & $S_{8} D_{\mathrm{IA}}=\sigma_{8}\left(\Omega_{m} / 0.3\right)^{\alpha} D_{\mathrm{IA}}$ \\
\hline MCCL non-tomo with marginalized systematics & $0.895_{-0.039}^{+0.054}$ \\
MCCL non-tomo with statistical uncertainty only & $0.881_{-0.053}^{+0.050}$ \\
MCCL non-tomo with marginalized systematics, $\alpha=0.6$ & $0.907_{-0.040}^{+0.047}$ \\
MCCL non-tomo, HALOFIT, no baryons & $0.880_{-0.038}^{+0.047}$ \\
IM3SHAPE non-tomo, $C_{\ell}$ analysis & $0.857_{-0.048}^{+0.063}$ \\
IM3SHAPE non-tomo, HALOFIT, no baryons, $C_{\ell}$ analysis & $0.846_{-0.054}^{+0.054}$ \\
DES Y1 cosmic shear fiducial (METACALIBRATION), tomographic (Troxel et al. 2017$)$ & $0.782_{-0.027}^{+0.027}$ \\
DES Y1 IM3SHAPE, tomographic (Troxel et al. 2017$)$ & $0.799_{-0.045}^{+0.048}$ \\
\hline \hline
\end{tabular}


data vector. First, we use a single tomographic bin, as opposed to four bins used in [74]. This results in weaker constraining power in our analysis, roughly by a factor of two. While no nontomographic constraints were measured in the main DES Y1 analysis, the Science Verification [66] analysis compared the fiducial 3-bin tomographic and nontomographic measurements. The constraining power was found to decrease roughly by a factor of two. We find a similar error scaling between our nontomographic MCCL analysis and the 4-bin tomography in DES Y1. Second, the choice of scale cuts were slightly different: the DES Y1 used scales $<7$ arcmin for $\xi_{+}$, which corresponds to $\ell>1500$. The range of $\xi_{-}$was restricted to large angular scales. The minimum scale was different in each redshift bin. We restrict our analysis to $\ell \in[200,950]$.

Detection and measurement of objects in the DES images is a part of the MCCL method. We use the $i$-band only for creating the source galaxy sample, and all five bands $(\operatorname{griz} Y)$ to obtain the $\mathrm{ABC}$ posterior on the parameters of the forward model. Our fiducial source sample contains 15,432,057 galaxies after applying the cuts described in Appendix E. This is similar to 22 million selected from the $r$-band only IM3SHAPE catalog in the main Y1 analysis [40,74]. The METACALIBRATION sample is larger and contains $\sim 34$ million objects using joint measurements from three bands. This results in a higher mean $n(z)$ from 4 tomographic bins with $\langle z\rangle \approx 0.67$ (based on Fig. 16 in [74]). This is higher than the $n(z)$ we obtained, which was $\langle\bar{z}\rangle=0.598 \pm 0.024$. The uncertainty on both mean redshift is close to that calculated for IM3SHAPE by [12], which ranged from $\sigma(\Delta z) \in(0.11,0.22)$ among tomographic bins. The error on the shear calibration is also close; [8] calculated $\sigma(m)=0.025$, while the spread of the calibration parameter $\eta$ (see Sec. IVA) in our ABC posterior is $\sigma(\eta)=0.022$.

Finally, we do not include marginalization over the intrinsic alignment amplitude and present a combination of $S_{8} D_{\mathrm{IA}}$. In the tomographic analysis of DES Y1 by [74], the uncertainty increased by $\sim 30 \%$ due to marginalization over wide IA prior. Marginalization over intrinsic alignment in the nontomographic analysis would lead to a more significant increase of the uncertainty on the cosmology parameters. Moreover, the DES analysis by [74] uses HALOFIT $[75,76]$ to model the nonlinear power spectrum and removes the dependence on the baryons by applying scale cuts. In our analysis, we use the HMCODE model by [64] and marginalize over a wide prior on baryonic corrections.

The tomographic DES Y1 analysis by [74] found $\sigma_{8}\left(\Omega_{m} / 0.3\right)^{0.5}=0.782_{-0.027}^{+0.027}$ with the METACALIBRATION data, and $\sigma_{8}\left(\Omega_{m} / 0.3\right)^{0.5}=0.799_{-0.045}^{+0.048}$ with the IM3SHAPE data. We investigate the main source of the difference between the MCCL and IM3SHAPE results by comparing the theory modeling, shear calibration, and $n(z)$ measurement, between the pipelines. We calculate constraints for the
MCCL $C_{\ell}$ and $n(z)$ using the HALOFIT [76] theory prediction, without marginalization of the strength of baryon effects, keeping the rest of the analysis configuration the same as for the main result. We find $S_{8} D_{\mathrm{IA}}=$ $0.880_{-0.038}^{+0.047}$ for that configuration, which is a small shift at the $0.5 \sigma$ level from the value obtained with HMCODE in the fiducial configuration.

We calculate the cosmological constraints from the IM3SHAPE shape catalog and the corresponding $n(z)$, with the likelihood analysis used for the MCCL result. To do it, we create a nontomographic source galaxy sample with a single $n(z)$ bin, by adding four tomographic $n(z)$, weighted by the corresponding effective galaxy densities [40][see] [for more details]. We add the redshift calibration shifts calculated by [12]. The mean redshift of this sample is $\bar{z}=0.591$, which is similar to the one obtained with MCCL. IM3SHAPE and MCCL use different galaxy selection, with roughly $70 \%$ galaxies overlapping between these two catalogs. The difference in the selection can be attributed to cuts on the PSF size, which is different in the two bands. Moreover, different bands were used: $r$-band for IM3SHAPE and $i$-band for MCCL, which results in different pixel noise.

We create shear maps with the IM3SHAPE catalog by pixelizing the shapes using Eq. (7.3) in [40] and calculate the $C_{\ell}$. We find that it largely agrees with the one obtained by MCCL, with the average difference of $3.5 \%$ on the $C_{\ell}$, which would correspond to $\approx 1.75 \%$ multiplicative shear bias $m$ if the $n(z)$ were identical. This level of difference is within our uncertainty on $\mathrm{m}$. We create the covariance matrix for the nontomographic IM3SHAPE $C_{\ell}$ using the same method as for the MCCL $C_{\ell}$, using the IM3SHAPE $n(z)$ and shape noise. We marginalize over the redshift and multiplicative shear errors by creating $30 C_{\ell}$ and $n(z)$ pairs, similarly as in the MCCL method. For each pair, we modify the $C_{\ell}$ to account for the shear calibration error drawn from the Gaussian prior with $\sigma=0.025$ [40], and a shifts drawn from a the prior on $\Delta z$ [12] for each redshift bin, during the creation of the nontomographic $n(z)$. With that configuration, we obtain $S_{8} D_{\mathrm{IA}}=0.857_{-0.048}^{+0.063}$. With HALOFIT, we obtain $S_{8} D_{\text {IA }}=0.846_{-0.045}^{+0.054}$. This is somewhat higher than the tomographic result in [74], at the level of $\approx 1 \sigma$, and lower than the MCCL (nontomographic) analysis. This indicates that the specific analysis choice of the DES Y1 data, using nontomographic $C_{\ell}$ in the range $\ell \in[200,950]$, gives somewhat higher $S_{8} D_{\mathrm{IA}}$ values than that of the tomographic, real space analysis in [74]. This level of differences is not unexpected, as selecting different scales for the analysis can cause shifts of the constraints within the statistical uncertainty.

\section{IMPLEMENTATION AND RUNTIME}

The integrated MCCL pipeline is designed to achieve a fast, integrated analysis. Our optimized implementation 
allows to run multiple full-area $i$-band simulations in the control loops framework within less than 48 hours. This process includes: simulating 30 full-area DES Y1 simulations from $\mathrm{ABC}$ posterior points, measurement of the $n(z)$ curves and shear calibration parameters, calculation of power spectra and covariance matrix, and calculation of the cosmological constraints. The simulation of a single DES Y1 survey area in the $i$-band takes about 2 hour on 400 cores. Several elements of the pipeline are precomputed: the $\mathrm{ABC}$ posterior on astrophysical and instrument parameters, the trained CNN for PSF estimation and the L-Picola simulations. From the above, the most time consuming part is the $\mathrm{ABC}$ posterior calculation, which takes several days on 1000 cores. The fast integrated analysis pipeline, which spans the analysis from image pixels to the cosmological constraints, allows for rapid testing of various parameter configurations and their direct impact on resulting cosmology measurement.

\section{CONCLUSIONS}

We present the application of the MCCL to a nontomographic cosmic shear reanalysis of the DES Y1 data. Using this method, we simultaneously measure the shear E-mode angular power spectrum $C_{\ell}$ and the redshift distribution $n(z)$ of the galaxy samples used for the shape measurements.

After giving an overview of the method, we discuss the specific implementation for this data set. In particular, we develop and apply detailed systematic maps for the DES Y1 coadds. We also model the PSF using deep learning, followed by an interpolation scheme. We then perform a first control loop to match the simulation to the real data and find a good match following an $\mathrm{ABC}$ inference for the simulation parameters. In the next control loop, we calibrate the shear measurement at the 1-point level and test it using a series of 2-point statistics. In the third loop, we perform a tolerance analysis for the cosmic shear measurement and derive statistical and systematic uncertainties for both $C_{\ell}$ and $n(z)$.

Given the resulting set of measurements for these two quantities and after unblinding, we derive cosmological parameter constraints and give an error budget. Including both statistical and systematic uncertainties, we find $S_{8} D_{\mathrm{IA}}=\sigma_{8}\left(\Omega_{m} / 0.3\right)^{0.5} D_{\mathrm{IA}}=0.895_{-0.039}^{+0.054}$, where $D_{\mathrm{IA}}=$ $1-0.11\left(A_{\mathrm{IA}}-1\right)$ is a factor that scales $\sigma_{8}$ according to the intrinsic alignment amplitude (see Sec. V B). We find that the systematic uncertainties contribute to the error budget on the level of $\approx 60 \%$ of the statistical error. Given that our nontomographic analysis does not lift the degeneracy between $S_{8}$ and $A_{\mathrm{IA}}$, a direct comparison with the earlier tomographic analysis [74] is not straightforward. Considering the degenerate product $S_{8} D_{\text {IA }}$ our results yield a somewhat higher value of this parameter than can be anticipated from this earlier analysis. To investigate the source of this difference we analyze the DES Y1 IM3SHAPE catalog using our choices of data vector (nontomographic $\left.C_{\ell} \in[200,950]\right)$ and the MCCL likelihood pipeline, using the HMCODE power spectrum. We find a similar nontomographic $n(z)$ between the MCCL and IM3SHAPE, as well as $C_{\ell}$ in agreement on the $3.5 \%$ level. The measurement of $S_{8} D_{\text {IA }}$ with IM3SHAPE with these settings is close to the MCCL results, and differs on $<1 \sigma$ level. This indicates that the specific analysis choice we made for the DES Y1 data yields a higher $S_{8} D_{\mathrm{IA}}$ result than expected from the tomographic analysis of [74]. The remaining difference can be attributed to different pixel noise between $r$ and $i$-bands, as well as differences in galaxy selection (see Sec. V G). Moreover, we find an additional difference in measured $S_{8} D_{\text {IA }}$ stemming from the use of the HMCODE instead of HALOFIT, on the level of $0.5 \sigma$.

The analysis presented here is the first end-to-end application of the MCCL method on a full data set and demonstrates the feasibility and accuracy of the method. In particular, the fast implementation and integrated nature of the MCCL pipeline offers a very short turnover time thus enabling the exploration, correction and quantification of systematics. This offers excellent prospects for the application of the MCCL to future weak lensing data sets. It can also be naturally extended to a tomographic configuration.

\section{ACKNOWLEDGMENTS}

We thank Joel Bergé, Chihway Chang and Lukas Gamper for crucial contributions to the development of UFIG and the MCCL method. We thank Janis Fluri for helpful conversations and help with deep learning aspect of PSF modeling. We thank Uwe Schmitt and Jarunan Panyasantisuk for informatics support. We acknowledge support by Grant No. 200021_169130 from the Swiss National Science Foundation. We acknowledge the support of Euler cluster by High Performance Computing Group from ETHZ Scientific IT Services, as well as the support of the Piz Daint cluster by the Swiss National Supercomputing Center (CSCS). Funding for the DES Projects has been provided by the U.S. Department of Energy, the U.S. National Science Foundation, the Ministry of Science and Education of Spain, the Science and Technology Facilities Council of the United Kingdom, the Higher Education Funding Council for England, the National Center for Supercomputing Applications at the University of Illinois at Urbana-Champaign, the Kavli Institute of Cosmological Physics at the University of Chicago, the Center for Cosmology and Astro-Particle Physics at the Ohio State University, the Mitchell Institute for Fundamental Physics and Astronomy at Texas A\&M University, Financiadora de Estudos e Projetos, Fundação Carlos Chagas Filho de Amparo à Pesquisa do Estado do Rio de Janeiro, Conselho Nacional de Desenvolvimento Científico e Tecnológico and the Ministério da Ciência, Tecnologia e Inovação, the Deutsche Forschungsgemeinschaft and the Collaborating Institutions in the Dark 
Energy Survey. The Collaborating Institutions are Argonne National Laboratory, the University of California at Santa Cruz, the University of Cambridge, Centro de Investigaciones Energéticas, Medioambientales y Tecnológicas-Madrid, the University of Chicago, University College London, the DESBrazil Consortium, the University of Edinburgh, the Eidgenössische Technische Hochschule (ETH) Zürich, Fermi National Accelerator Laboratory, the University of Illinois at Urbana-Champaign, the Institut de Ciències de l'Espai (IEEC/CSIC), the Institut de Física d'Altes Energies, Lawrence Berkeley National Laboratory, the LudwigMaximilians Universität München and the associated Excellence Cluster Universe, the University of Michigan, the National Optical Astronomy Observatory, the University of Nottingham, The Ohio State University, the University of Pennsylvania, the University of Portsmouth, SLAC National Accelerator Laboratory, Stanford University, the University of Sussex, Texas A\&M University, and the OzDES Membership Consortium. Based in part on observations at Cerro Tololo Inter-American Observatory, National Optical Astronomy Observatory, which is operated by the Association of Universities for Research in Astronomy (AURA) under a cooperative agreement with the National Science Foundation. The DES data management system is supported by the National Science Foundation under Grants No, AST-1138766 and No. AST-1536171. The DES participants from Spanish institutions are partially supported by MINECO under Grants No. AYA2015-71825, No. ESP201566861, No. FPA2015-68048, No. SEV-2016-0588, No. SEV2016-0597, and No. MDM-2015-0509, some of which include ERDF funds from the European Union. I. F. A. E. is partially funded by the CERCA program of the Generalitat de Catalunya. Research leading to these results has received funding from the European Research Council under the European Union's Seventh Framework Program (FP7/ 2007-2013) including ERC Grant agreements No. 240672, No. 291329, and No. 306478. We acknowledge support from the Brazilian Instituto Nacional de Ciência e Tecnologia (INCT) e-Universe (CNPq grant No. 465376/2014-2). This manuscript has been authored by Fermi Research Alliance, LLC under Contract No. DE-AC02-07CH11359 with the U.S. Department of Energy, Office of Science, Office of High Energy Physics. The United States Government retains and the publisher, by accepting the article for publication, acknowledges that the United States Government retains a non-exclusive, paid-up, irrevocable, world-wide license to publish or reproduce the published form of this manuscript, or allow others to do so, for United States Government purposes.

\section{APPENDIX A: BLINDING SCHEME}

We adopt a blinding scheme to avoid confirmation biases. For this purpose, we introduce a blinding normalization and tilt in the EE weak lensing power spectrum $C_{\ell}$. These two parameters are kept blinded until the results pass predefined tests.
To blind a $C_{\ell}$, we transform it the following way:

$$
C_{\ell}^{\text {blinded }}=C_{\ell}\left(c_{0}+\mu\left(\ell / \ell_{0}-1\right)\right)
$$

where $c_{0}$ and $\mu$ are blinding factors, and $\ell_{0}=500$. The blinding factors were unknown to us, and were drawn from a uniform distribution with ranges $c_{0} \in[0.8,1.2]$ and $\mu \in[-0.3,0.3]$. This configuration effectively blinds the $C_{\ell}$ amplitude in $40 \%$ range, and adds tilt of up to $30 \%$. This blinding scheme was different than the one used in [74], where shear values are multiplied by a factor of between 0.9 and 1.1. The allowed modification of the shear 2-pt function is similar for both strategies.

In Sec. VE we list a set of conditions that need to be satisfied before unblinding. We consider these conditions to be satisfied, below we summarize the sections in the paper which correspond to each of them.

(Q1) The histograms of relevant quantities are described in Sec. III D and Fig. 4. The histograms obtained from the DES data are contained within the ones from simulations for most of their range, except for tails on several panels. As these tails contain relatively few objects compared to the bulk of the distribution, we do not expect these differences to impact the result. We find a small difference in the FLUX_RADIUS parameter.

$(Q 2)$ The recovery of the input cosmology for fiducial simulation is satisfactory, as described in Appendix F.

(Q3) According to the systematics model presented in Sec. IV A 2, the contribution of remaining systematic maps due to PSF leakage and PSF ellipticity and size modeling errors should not significantly affect the measurement.

(Q4) We do not detect significant B-mode (see Sec. IVA 3, which satisfies this condition.

(Q5) We test one extension to the model in Sec. IV C. We checked if adding scatter to the Sérsic index of blue galaxies changes the measurement. We found that neither the shear calibration parameters nor the $n(z)$ change significantly.

The following actions were taken after unblinding. We found and corrected a mistake in calculation of the GI contribution to the power spectrum. This change results in an increase in measured $S_{8} D_{\mathrm{IA}}$ by $\approx 0.5 \sigma$. We also found two minor mistakes in the calculation of covariance matrix concerning the subtraction of the mean of the convergence maps and rotation of the shear for some of the ten patches. As the covariance matrix is dominated by shape noise, we found that these mistakes did not noticeably affect the final result.

\section{APPENDIX B: POWER SPECTRUM CALCULATION}

We create pixelized shear maps from the source galaxy sample by averaging the shapes of all galaxies in each 
pixel, with no weighting of shapes applied. We use the HEALPix scheme to create the maps. We compute all spherical harmonic power spectra using the publicly available code POLSPICE [77] [49-51]. In order to estimate the values of the maximal angular scale used by POLSPICE, $\theta_{\max }$, and the apodization parameter $\theta_{\mathrm{FWHM}}$, we follow the method outlined in $[55,56]$. From this stage onwards, we follow the blinding scheme described in Appendix A. Calculated $C_{\ell}$ were averaged into 15 linearly spaced bins in the range between $\ell_{\min }=200$ and $\ell_{\max }=950$.

For the ellipticity maps, the mean of the map, corresponding to $\ell=0$, is subtracted before passing it to POLSPICE. We process scalar quantities, such as PSF size, in the form of fractional difference maps, $(x-\bar{x}) /(\bar{x})$, where $\bar{x}$ is the mean of the map.

We subtract the contribution of the noise from auto power spectra. The noise contribution is calculated by taking a mean of $100 C_{\ell}$ calculated from maps with removed signal. For scalar maps, the positions of the stars/galaxies were randomly permuted. For spin-2 ellipticities, the signal was removed by randomly rotating the shapes. This procedure still contains a small contribution of the shear to the noise estimate, which slightly increases the variance. This increase is extremely small and does not make any effective difference to the noise model.

To account for variable number of galaxies in each pixel, we compute the power spectrum using an additional weight map. The weight map corresponds to inverse variance weights and is simply a map of source galaxy number counts.

\section{APPENDIX C: PSF ESTIMATION AND MODELING}

As described in Sec. III, we rely on the approach presented by [42] to estimate and model the PSF. However, we modify the parametrization of the PSF model, parameter estimation details, and neural network training strategy. We detail and motivate these changes in this section. We also present the details of the interpolation method on coadded images.

\section{PSF model and parameter estimation}

The updated model contains a modified base profile. We use a mixture of two Moffat profiles with $\beta_{1}=2$ and $\beta_{2}=5$. Parameter $f_{\beta} \in[0,1]$ controls the fraction of the photons sampled from the first profile. Note that in [42] this parameter was fixed and labeled $\gamma$. We also modify the perturbation of the photon position associated with the kurtosis operation. The position of photons $\theta_{i}$ is perturbed by $\delta \theta_{i}$ in the following way:

$$
\begin{gathered}
\delta \theta_{i}=k \cdot\|\theta\|_{2}^{2} \cdot \beta^{-1} \cdot \theta_{i} \cdot k_{s}, \\
k_{s}=\exp \left[-0.5\|\theta\|_{2}^{2} /\left(0.2 \beta^{2}\right)\right] .
\end{gathered}
$$

where $k$ is the kurtosis parameter and $k_{s}$ is a suppression factor dependent on the profile. This factor prevents extremely large displacements for photons in the wings of the profile. In total, the model has 11 parameters: centroid positions $x, y$, full width half maximum (FWHM) $r_{h}$, ellipticities $e_{1}, e_{2}$, flexions $f_{1}, f_{2}, g_{1}, g_{2}$, flux ratio between two Moffat components $f_{\beta}$ and kurtosis $k$. See [42] for the definitions of these parameters. The centroid positions are not used later in any way. We found that it is difficult to measure the kurtosis parameter, as it does not modify the profile in a significant way within the prior range we considered.

We used image cutouts of size $19 \times 19$ pixels. To generate the training data, we draw magnitudes between 17 and 22, gain values between 3 and $5.5 e^{-} / \mathrm{ADU}$, the number of exposures between 1 and 9 and we use a magnitude zero point of 30 , which is the value of the DES Y1 data. During the training, we add Gaussian background noise with standard deviations sampled from the interval OADU to $10 \mathrm{ADU}$ on the fly.

We train the CNN using a different objective function than [42], the likelihood loss, similarly to [78]. We use single parameter variances instead of a full covariance matrix. Another modification to the cost function is the use of averaging of multiple noise realizations before taking the square of the residuals, which greatly reduces the bias of the recovered parameters. The cost $L$ is

$$
L=\sum_{i \in N_{b}} \sum_{p \in N_{p}}\left[\left\langle\frac{\hat{\theta}_{i, n}^{p}-\theta_{i, n}^{p}}{\sigma_{p}}\right\rangle_{N_{n}}^{2}+\log \left\langle\sigma_{p}\right\rangle_{N_{n}}^{2}\right],
$$

where $N_{b}=2048$ is the number of images in a batch, $N_{p}=11$ is the number of parameters in the model, $N_{n}=64$ is the number of noise realizations per parameter set, $\hat{\theta}_{i, n}^{p}$ is the network's prediction, $\theta_{i, n}^{p}$ is the true parameter and $\sigma_{p}$ is the uncertainty of parameter $p$, the logarithm of which is also predicted by the network. Angular brackets denote averaging over the number of noise realizations $N_{n}$. To train using this loss function, we generated a training set consisting of $10^{9}$ star images samples using latin hypercube sampling in ranges of magnitude, gain and number of exposures mentioned above. The remaining parameters had the following span: $r_{h} \in[2.5,5], e_{1}, e_{2}, f_{1}, f_{2}, g_{1}, g_{2} \in[-0.1,0.1], k \in$ $[-0.5,0.5], x, y \in[9,10], f_{\beta} \in[0 ., 1$.$] .$

In the training process we progressively increase the noise levels and decreased the learning rate. We train the network with no added Gaussian noise (the training images already contained Poisson noise) on 100'000 iterations, then we gradually increase the noise level until it reaches $\sigma_{n}=10$ at $200 ' 000$ iteration. The learning rate is initiated at $l_{r}=0.001$, decreased at steps $\left[5 \times 10^{4}, 10^{5}, 2 \times 10^{5}\right]$ to $l_{r}=\left[10^{-4}, 5 \times 10^{-5}, 2 \times 10^{-5}\right]$, respectively. We stored the 
network that gave us the best loss value, which happened at iteration 562,186.

\section{PSF interpolation}

We interpolate the PSF across the coadded images. We model the PSF surface for each of the parameters, except the $x, y$ positions, using a Chebyshev polynomial basis of maximum order 4 . The basis also includes information about the tiling pattern coming from the coadds. We create a pseudo-Vandermonde matrix $\Phi_{i j k}$ of degree 4 for sample points $x$ and $y$ in the coadd pixel coordinate system

$$
\Phi_{i j k}(x, y)=T_{i}(x) T_{j}(y) W_{k}(x, y),
$$

where $T_{i}$ is the Chebyshev polynomial of order $i$ and $W_{k}$ is a weight corresponding to exposure $k$. If an object at position $(x, y)$ was recorded by exposure $k$, then the weight is nonzero, and has a value $W_{k}(x, y)=1 / N_{x, y}^{e}$, where $N_{x, y}^{e}$ is the total number of exposures contributing to the image at position $(x, y)$. This model assumes equal-weighted contributions to the PSF parameters from each exposure, which may not be true in general; we do not attempt to make a more detailed model of this process, leaving that to future work. The basis in Eq. (C4) has total of $4^{2} N_{\text {exp }}$ parameters for each coadd, where $N_{\text {exp }}$ is the maximum number of exposures for that coadd. We fit a surface for each parameter iteratively, using a robust technique based on $\sigma$-clipping. After the initial fit, the $3 \sigma$ outliers in $r_{p}, e_{1}, e_{2}$
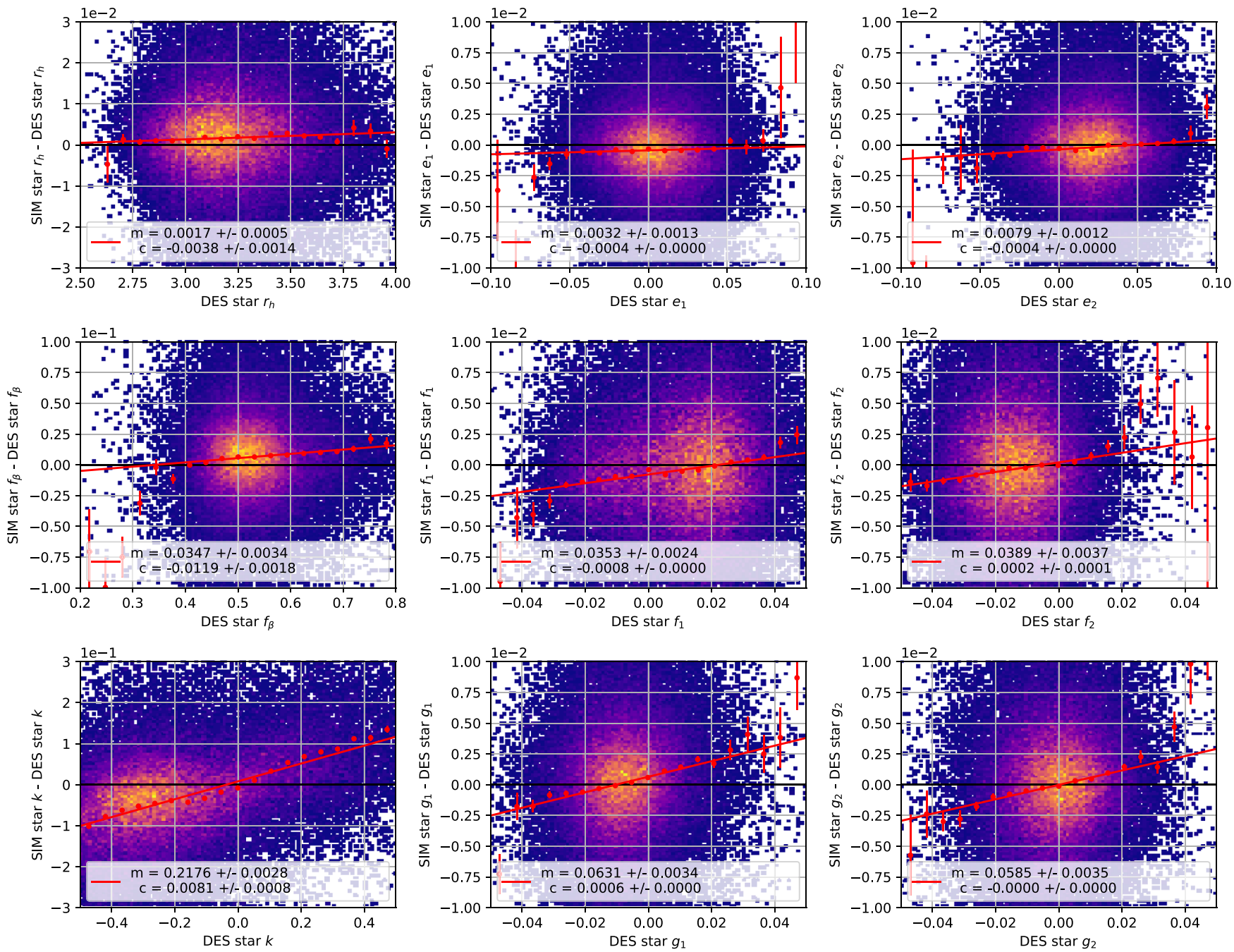

FIG. 10. Accuracy of the PSF measurement and interpolation for PSF model parameters: size $r_{p}$, ellipticities $e_{i}$ flexions $f_{i}, g_{i}$, flux ratio of profile components $f_{\beta}$, and kurtosis $k$. Each panel shows the residual between the interpolated value and the true value of the parameter as a function of the true value. Red points correspond to the mean of the residual in bins, and the error bars represent the standard deviation. Red lines show the line fit to these points, with parameters stated in the legend, where $m, c$ are the slope and intercept, respectively. 
are removed, and the fit is performed again until convergence, and at maximum 10 times. The final model is stored. The iterative fitting procedure makes the model more robust to stars with unusual parameters, extreme measurement errors, stars blended with galaxies and false positives in the Gaia catalog. Before fitting, we exclude $15 \%$ of stars randomly to create a validation star sample, used later for testing the PSF interpolation.

Figure 10 shows the accuracy of the PSF modeling from our fiducial simulation. We plot the difference between PSF parameters estimated from $\sim 60,000$ validation stars in DES and simulations at the exact same positions. The errors in predictions are shown as a function of the parameter estimated for the DES star. The red points show the means of residuals in bins spanning the range shown, and the error bars correspond to error on the mean. The red line shows a linear fit to these points. We notice that the PSF recovery is generally good; small biases exist for size, ellipticities and flexions, while the recovery is worse for kurtosis and profile flux ratio. Similar level errors is observed when the full sample of stars is used, including the ones used for PSF estimation, as well as in simulations, when the estimated PSF model parameters are compared with the true input. As the overall error in E-mode reconstruction in simulations is satisfactory with respect to the requirements (see Appendix F), we conclude that these differences do not have a significant impact on the shear calibration.

\section{APPENDIX D: ABC ANALYSIS}

\section{Variable parameters and priors}

In this section, we present more details on the parameters we vary during the $\mathrm{ABC}$ analysis and specify the priors used the sample the 35-dimensional parameter space. As described in Sec. III D, the parameters we vary can be divided into six groups, which are listed below. The combined prior is a product of the individual priors. Table II summarizes the information presented in this section.

(1) Galaxy luminosity function parameters. These parameters are referred to as $a_{M}, b_{M}, a_{\phi}, b_{\phi}$ in [21] for the blue and the red population, respectively. They control the redshift evolution of the luminosity functions from which we sample the galaxy population. We use a modified version of the prior specified in [21], which was sampled with COSMOHAMMER using data given in [79]. Since this prior is well described by a multivariate normal distribution, we use the mean and covariance matrix computed from the COSMOHAMMER samples used by [21] to generate the new prior data for this work, which simplifies the sampling. We retain the enlarged prior volumes used in [21] for $b_{\phi}$. However, we reduce the enlargement for blue galaxies by multiplying each sample with a random number between 0.5 and 2.5 (instead of 0.5 and 4, as in [21]). Furthermore, we decided to limit the range of $b_{\phi}$ to the interval $[0,0.0075]$ for blue galaxies and to [0, 0.0175] for red galaxies. If a sample does not fall within these boundaries, we redraw. These modification were inspired by earlier $\mathrm{ABC}$ analyses, they are effectively a by-hand application of the $\mathrm{ABC}$ algorithm proposed by [80].

(2) Galaxy SED parameters. The parameters $\alpha_{i, 0}$ (at redshift $z=0$ ) and $\alpha_{i, 1}$ (at redshift $z=1$ ) control the Dirichlet distributions, from which we sample the template coefficients used to assign restframe SEDs to galaxies, see [21]. We retain the prior used by [21] for this work.

(3) Galaxy size parameters. The parameters $a_{\mu}, b_{\mu}, \sigma_{\text {phys }}$ from [21] set our model for the distribution of intrinsic galaxy sizes. As for the galaxy luminosity function parameters, we sample this part of parameter space using a multivariate normal distribution with the mean and covariance matrix computed from the corresponding COSMOHAMMER samples used by [21]. Since $\sigma_{\text {phys }}$ denotes a standard deviation, this parameter cannot be negative, therefore, we redraw if this happens. Furthermore, as was done in [21], we relax the prior on $b_{\mu}$. Here, we use a uniform prior ranging from 0.8 to 1.4 .

(4) Sérsic index for blue galaxies. We sample $n_{\text {blue }}$ from a uniform prior extending from 0.5 to 1.5 .

(5) Intrinsic ellipticity distribution for galaxies. The uniform prior for $e_{\text {ratio }}$ ranges from 0.3 to 0.6 and the uniform prior for $e_{\text {sum }}$ from 2 to 5 .

(6) Background noise scale. This parameter scales the background level noise globally for all simulated images. We place a uniform prior extending from 1.06 to 1.09 on this parameter.

\section{Distance metrics}

We use the five distance metrics listed below to infer our $\mathrm{ABC}$ posterior. As described in Sec. III D, we evaluate all samples on 20 DES tiles and average the individual values of the distance metrics to reduce cosmic variance.

(1) Magnitude histogram distance. This distance metric ensures that our posterior simulations match the DES data in terms of galaxy number counts and $r$-band magnitude distributions. It is computed by subtracting the binned $r$-band magnitude distribution of the DES lensing galaxies from the magnitude distribution of the simulated lensing galaxies and summing up the absolute differences, see [21]. This distance metric is averaged by stacking the histograms from the individual tiles and averaging the bin entries. The distance metric is then evaluated on the averaged histograms.

(2) MMD distance for magnitudes, sizes and ellipticity. We compute a 11-dimensional MMD distance 
TABLE II. Variable parameters and priors for the ABC analysis. See Sec. III A, Appendix D 1 as well as [21] for more information. The values of $\sigma^{2}$ for the multivariate normal distributions correspond to the diagonal element of the full covariance matrix.

\begin{tabular}{|c|c|c|c|}
\hline & Parameter & Meaning & Prior \\
\hline \multirow[t]{8}{*}{$\begin{array}{l}\text { Luminosity } \\
\text { functions }\end{array}$} & $a_{M}^{\text {blue }}$ & $\begin{array}{l}\text { Slope of the redshift of evolution of the parameter } M_{*} \text { in } \\
\text { the Schechter luminosity function for blue galaxies }\end{array}$ & $\begin{array}{l}\text { Multivariate normal, } \mu=-9.43 \times 10^{-1} \\
\sigma^{2}=2.09 \times 10^{-1}\end{array}$ \\
\hline & $b_{M}^{\text {blue }}$ & $\begin{array}{l}\text { Intercept of the redshift of evolution of the parameter } M_{*} \\
\text { in the Schechter luminosity function for blue galaxies }\end{array}$ & $\begin{array}{l}\text { Multivariate normal, } \mu=-2.04 \times 10^{1} \\
\sigma^{2}=8.33 \times 10^{-2}\end{array}$ \\
\hline & $a_{M}^{\mathrm{red}}$ & $\begin{array}{l}\text { Slope of the redshift of evolution of the parameter } M_{*} \text { in } \\
\text { the Schechter luminosity function for red galaxies }\end{array}$ & $\begin{array}{l}\text { Multivariate normal, } \mu=-7.07 \times 10^{-1} \\
\sigma^{2}=1.35 \times 10^{-1}\end{array}$ \\
\hline & $b_{M}^{\mathrm{red}}$ & $\begin{array}{l}\text { Intercept of the redshift of evolution of the parameter } M_{*} \\
\text { in the Schechter luminosity function for red galaxies }\end{array}$ & $\begin{array}{l}\text { Multivariate normal, } \mu=-2.04 \times 10^{1} \\
\sigma^{2}=7.46 \times 10^{-2}\end{array}$ \\
\hline & $a_{\phi}^{\text {blue }}$ & $\begin{array}{l}\text { Decay constant of the redshift of evolution of the } \\
\text { parameter } \phi_{*} \text { in the Schechter luminosity function for } \\
\text { blue galaxies }\end{array}$ & $\begin{array}{l}\text { Multivariate normal, } \mu=-1.17 \times 10^{-1}, \\
\sigma^{2}=3.49 \times 10^{-2}\end{array}$ \\
\hline & $b_{\phi}^{\text {blue }}$ & $\begin{array}{l}\text { Amplitude of the redshift of evolution of the parameter } \phi_{*} \\
\text { in the Schechter luminosity function for blue galaxies }\end{array}$ & $\begin{array}{l}\text { Multivariate normal, } \mu=3.76 \times 10^{-3} \\
\sigma^{2}=2.29 \times 10^{-7}, \text { multiplied by } \\
\text { random variate from }[0.5,2.5] \\
\text { bounded to }\left[0,7.5 \times 10^{-3}\right]\end{array}$ \\
\hline & $a_{\phi}^{\mathrm{red}}$ & $\begin{array}{l}\text { Decay constant of the redshift of evolution of the } \\
\text { parameter } \phi_{*} \text { in the Schechter luminosity function for } \\
\text { red galaxies }\end{array}$ & $\begin{array}{l}\text { Multivariate normal, } \mu=-8.96 \times 10^{-1} \text {, } \\
\sigma^{2}=2.47 \times 10^{-1}\end{array}$ \\
\hline & $b_{\phi}^{\mathrm{red}}$ & $\begin{array}{l}\text { Amplitude of the redshift of evolution of the parameter } \phi_{*} \\
\text { in the Schechter luminosity function for red galaxies }\end{array}$ & $\begin{array}{l}\text { Multivariate normal, } \mu=3.91 \times 10^{-3} \\
\sigma^{2}=1.14 \times 10^{-6}, \text { multiplied by by } \\
\text { random variate from }[0.5,4], \text { bounded } \\
\text { to }\left[0,1.75 \times 10^{-2}\right]\end{array}$ \\
\hline \multirow[t]{3}{*}{ Galaxy sizes } & $a_{\mu}$ & $\begin{array}{l}\text { Slope of the evolution of the average intrinsic physical } \\
\text { size of galaxies with absolute magnitude }\end{array}$ & $\begin{array}{l}\text { Multivariate normal, } \mu=-0.24 \times 10^{0} \\
\sigma^{2}=3.31 \times 10^{-6}\end{array}$ \\
\hline & $b_{\mu}$ & $\begin{array}{l}\text { Intercept of the evolution of the average intrinsic physical } \\
\text { size of galaxies with absolute magnitude }\end{array}$ & Uniform in $[0.8,1.4]$ \\
\hline & $\sigma_{\text {phys }}$ & $\begin{array}{l}\text { Standard deviation of the normal distribution we use to } \\
\text { sample intrinsic physical galaxy sizes }\end{array}$ & $\begin{array}{l}\text { Normal multivariate, } \mu=0.57 \times 10^{0} \\
\sigma^{2}=6.36 \times 10^{-6}, \text { bounded } \\
\text { to positive values }\end{array}$ \\
\hline Galaxy profiles & $n_{\text {blue }}$ & Sérsic index of blue galaxies & Uniform in $[0.5,1.5]$ \\
\hline $\begin{array}{l}\text { Galaxy } \\
\text { ellipticities }\end{array}$ & $\begin{array}{l}e_{\text {ratio }} \\
e_{\text {sum }}\end{array}$ & $\begin{array}{l}\text { Parameters controlling the beta distribution from which } \\
\text { we sample intrinsic galaxy ellipticities }\end{array}$ & $\begin{array}{l}\text { Uniform in }[0.3,0.6] \\
\text { Uniform in }[2,5]\end{array}$ \\
\hline $\begin{array}{l}\text { Background } \\
\text { noise }\end{array}$ & $s_{\text {bkg }}$ & $\begin{array}{l}\text { Scale factor for the background noise level in the } \\
\text { simulations }\end{array}$ & Uniform in $[1.06,1.09]$ \\
\hline \multirow[t]{4}{*}{$\begin{array}{l}\text { Template } \\
\text { coefficients }\end{array}$} & $\alpha_{i, 0}^{\text {blue }}$ & $\begin{array}{l}\text { Concentration parameters of the Dirichlet distribution at } \\
\text { redshift } z=0 \text { from which the template coefficients for } \\
\text { blue galaxies are sampled, } i=1, \ldots, 5\end{array}$ & \multirow{4}{*}{$\begin{array}{l}\text { Dirichlet distributions with equal concen- } \\
\text { tration parameters; the sums of the con- } \\
\text { centration parameters are uniformly } \\
\text { distributed in }[5,15]\end{array}$} \\
\hline & $\alpha_{i, 1}^{\text {blue }}$ & $\begin{array}{l}\text { Concentration parameters of the Dirichlet distribution at } \\
\text { redshift } z=1 \text { from which the template coefficients for } \\
\text { blue galaxies are sampled, } i=1, \ldots, 5\end{array}$ & \\
\hline & $\alpha_{i, 0}^{\mathrm{red}}$ & $\begin{array}{l}\text { Concentration parameters of the Dirichlet distribution at } \\
\text { redshift } z=0 \text { from which the template coefficients for } \\
\text { red galaxies are sampled, } i=1, \ldots, 5\end{array}$ & \\
\hline & $\alpha_{i, 1}^{\mathrm{red}}$ & $\begin{array}{l}\text { Concentration parameters of the Dirichlet distribution at } \\
\text { redshift } z=1 \text { from which the template coefficients for } \\
\text { red galaxies are sampled, } i=1, \ldots, 5\end{array}$ & \\
\hline
\end{tabular}

(maximum mean discrepancy, see [21,81]) using the measured magnitudes and sizes in the five DES filter bands as well as the measured ellipticity in the $r$-band. This distance metric selects samples which match the DES data in terms of colors, magnitude-size-distributions and $i$-band ellipticities. To evaluate the MMD distance, we use the lensing galaxies. The values of the distance metrics computed from the individual DES tiles are directly averaged to obtain a mean value for one sample. 
(3) VVDS-Wide MMD distance. As was done in [21], we use the VVDS-Wide spectroscopic sample to compute a two-dimensional MMD distance using measured $i$-band magnitudes as well as redshifts. The VVDS-Wide sample is purely magnitude-limited in the $i$-band, such that we can easily emulate that sample from our simulations and use it to tighten our constraints on the redshift distribution. To average this distance metric over multiple DES tiles, we directly average the distance values obtained from the individual coadded images.

(4) Galaxy profile distance. To constrain the Sérsic index for blue galaxies, we use a distance metric based on the light distributions of our lensing galaxies in the $r$-band. We cut out $21 \times 21$ pixel stamps of these objects and compute the pixel-wise mean, which yields the average lensing galaxy profile for one tile. We normalize the profile to

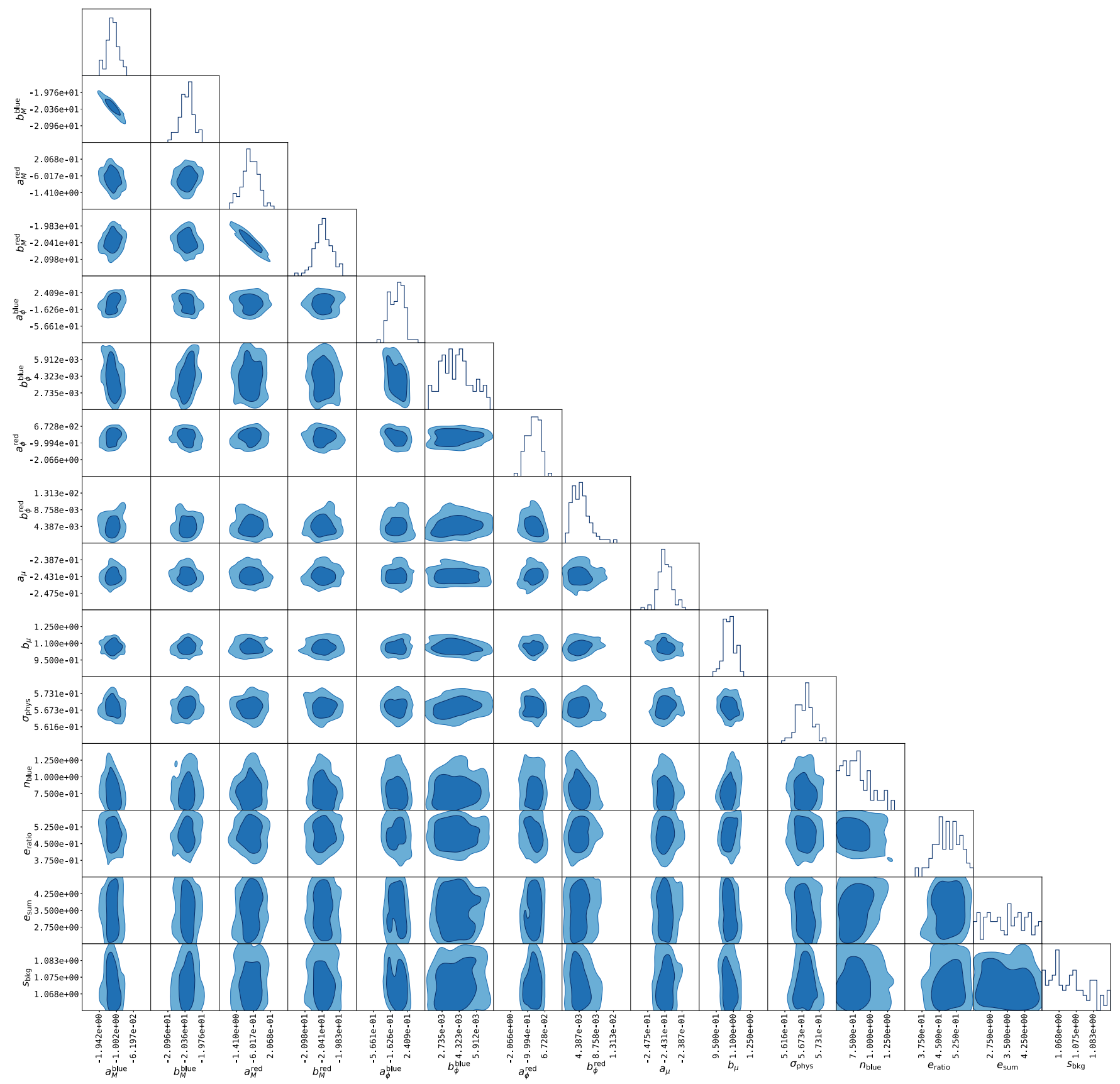

FIG. 11. Posterior distribution on the parameters of the forward model. This distribution was found using ABC. Parameters of the template coefficients are not shown. The shaded regions show $68 \%$ and $95 \%$ confidence intervals. 

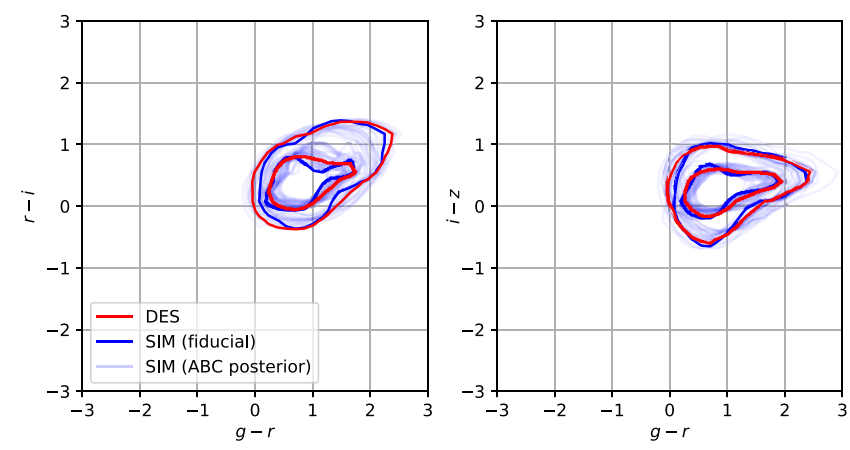

FIG. 12. Colors of galaxies in the DES (red) and fiducial UFig (blue) samples. The contours signify $65 \%$ and $95 \%$ confidence limits. The light blue contours correspond to the $30 \mathrm{ABC}$ posterior points. These simulations are made during the $\mathrm{ABC}$ run; they use griz bands and 20 randomly selected tiles (see also Sec. III D).

have a maximum pixel flux of 1 and then compute the pixel-wise sum of the squared difference of the profile from the simulated data and the one obtained from DES data. To average this distance metric over multiple tiles, we first average the mean profiles extracted from the individual tiles and then evaluate the distance metric.

(5) Background level distance. To constrain the parameter scaling the background level of the simulated images, we use histograms of pixel values between -10 and 10ADU, which characterize the background noise. We compute these histograms from the DES data and from the simulations. We then subtract the histogram computed from DES from the histogram obtained from a simulated image and sum up the absolute differences in the bin entries to obtain a distance metric. To average this distance metric over multiple tiles, we stack the corresponding histograms and average the bin entries. The distance metric is then evaluated on the averaged histograms.

\section{3. $\mathrm{ABC}$ posterior}

Concerning the thresholding prescription, we adapt the method given by [80]. We scale all distance metrics to cover similar numerical ranges by dividing by the corresponding 10th percentiles. Furthermore, we downweight distances number 1 and 5 by a factor of 0.2 , which results in tighter constraints on $n(z)$ and the size and ellipticity distributions. We then combine all five distance metrics into a single value by taking the maximum. This combined distance metric is subsequently used to select a number of best samples.

The $\mathrm{ABC}$ posterior on 15 parameters in the forward model is shown in Fig. 11. The remaining 20 parameters corresponding to the template coefficients at redshifts $z=0$ and $z=1$ for red and blue galaxies are not shown here. We summarize the information about galaxy colors in
Figure 12. This figure shows the histogram of galaxy $g-r$, $r-z$ and $i-z$ colors. The red contours correspond to the DES survey sample, the dark blue to the UFig simulation from the fiducial $\mathrm{ABC}$ posterior point, and the light blue to the simulations from the 30 posterior points used throughout the analysis. There is a good match between the colors in the survey data and the simulations.

\section{APPENDIX E: GALAXY SAMPLE SELECTION}

The minimum and maximum galaxy-to-PSF size ratios are set to $r_{g} / r_{h}^{p}>0.75$ and $r_{g} / r_{h}^{p}<100$, respectively, where

$$
r_{g}=\sqrt{\left(M_{00}+M_{11}\right) \cdot 2 \cdot \log (2)}
$$

is the measured galaxy size [20], and the elements of the moment matrix $M$ are the SEXTRACTOR windowed moments $M_{00}=\mathrm{X} 2 \mathrm{WIN}$ IMAGE and $M_{11}=\mathrm{Y} 2 \mathrm{WIN}$ IMAGE.

The minimum signal-to-noise ratio to $S / N>10$, where $S / N=$ FLUXAUTO/FLUXERRAUTO. We require the objects in the Galaxy and PSF sample to have SEXTRACTOR flags FLAGS $=0$ or FLAGS $=16$. This ensures the removal of blended objects. We find that excluding objects with FLAGS=16 set to 1 was causing large selection biases on the shape. This is due to FLAGS $=16$ parameter being affected by the row-by-row scanning strategy employed by SEXTRACTOR. We additionally remove all galaxies from the source galaxy sample that had sizes outside the range $r_{g} \in[2,20]$ and ellipticities of the SEXTRACTOR windowed moments $\left|e_{M}\right|>1$. This ellipticity is simply calculated as $e_{M, 1}=\left(M_{00}-M_{11}\right) /$ $\left(M_{00}+M_{11}\right)$ and $e_{M, 2}=\left(2 M_{01}\right) /\left(M_{00}+M_{11}\right)$. We also removed galaxies for which the PSF prediction is unreliable, with the criterion: $r_{h} \in[2,12]$ and $\left|e_{\mathrm{PSF}}\right|<0.5$.

We remove all objects which lie on the border between chip images. We require that there are no breaks in the exposure maps (see Sec. III C) inside the galaxy's postage stamp of size 20 pixels. We expect the images lying on the boarder to have unreliable PSF models.

We remove all objects in areas covered by less than 3 exposures. We find that this cut greatly improves the B-mode statistics. This is due to the fact that a lot of these objects lie in areas between chips. These areas can have an unreliable PSF model. This cut removes around 25\% galaxies.

The analysis used 3373 tiles associated with the Y1A1 tag. However, we remove 18 tiles for which the SEXTRACTOR run or PSF estimation was consistently failing. These tiles were: DES0001-5705, DES0319-6456, DES0030-4331, DES2019-5957, DES2335-5705, DES2008-5248, DES0346-6456, DES0347-4123, DES0339-6039, DES2225-5957, DES0622-5248, DES0434-3957, DES2248-4706, DES2240-4623, DES2244-4706, DES0044-4123, DES0620-5331, DES2312-5123. 
The SEXTRACTOR parameters are

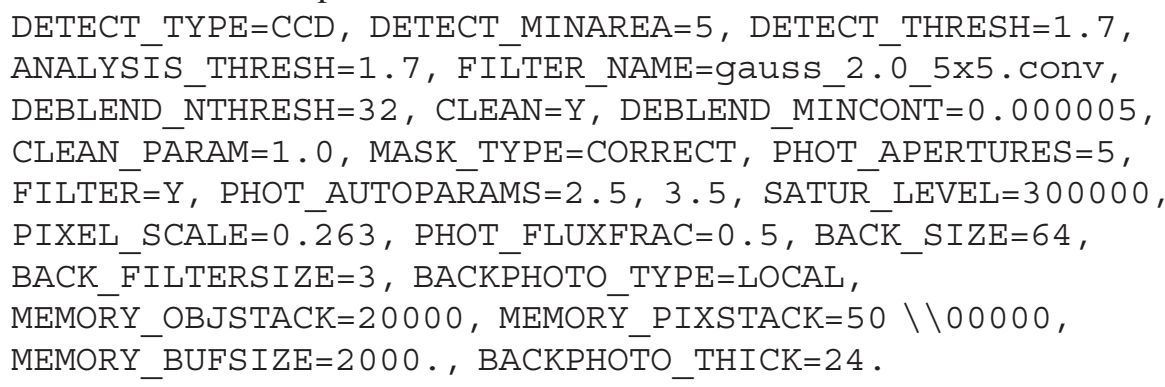

\section{APPENDIX F: SIMULATION INTERNAL TEST}

We perform an end-to-end test of the analysis pipeline to determine if we can recover the input cosmological parameters. We simulate a Gaussian shear field using Synfast, a part of HEALPy package, with the input power spectrum corresponding to the parameters $h=0.7, \Omega_{b}=0.05, \Omega_{m}=0.3, \sigma_{8}=0.8$, and an $n(z)$ of the fiducial survey. We run another 30 simulations with the fiducial survey parameters (see Sec. III D), changing only the random seeds in UFig. The input shear map and systematic maps are the same, while pixel noise, galaxy positions and parameters are randomly drawn from 30 different seeds. We analyze these 30 surveys separately in the exact same way as the DES data and apply the calibration parameters from the fiducial survey. Finally, we average the power spectra after noise correction. This allows us to reduce the statistical uncertainty of the power spectrum measurement. Left and right panels on Fig. 13 show the average EE and BB $C_{\ell}$. The true $C_{\ell}$ is shown with magenta line. The blue line shows the $C_{\ell}$ obtained with estimated PSF parameters used as an input for shear calibration. The cyan line shows the mean $C_{\ell}$ calculated using the true PSF parameters. This is the best-case scenario, when the PSF information is perfectly known. Error bars on the lines correspond to the errors on the mean $C_{\ell}$ and they exclude the cosmic variance. The light blue band corresponds to the $1 \sigma$ errors for a single survey, and are taken from the covariance matrix diagonal, similarly to Fig. 5.

The recovery of the power spectrum is generally good for the case when true PSF parameters were used in Eq. (1). We notice, however, a slight error on the recovered mean $C_{\ell}$ when the estimated PSF parameters are used. To examine the impact of that error, we calculated cosmological constraints using the mean $C_{\ell}$ of multiple realizations of the UFig full simulations. We averaged the $30 C_{\ell}$ and passed it as an input to the likelihood analysis, the same way as for the main result presented in Sec. V, including the same covariance matrix. No baryons or intrinsic alignments were used in this test. The left panel of Fig. 14 presents the $\sigma_{8}-\Omega_{m}$ constraints for the average $C_{\ell}$. The input cosmology is marked with a star. These constrains do not include marginalization over the systematic uncertainty from $n(z)$ and shear calibration. The constraint lies within $0.5 \sigma$ away from the input. The difference would be even less significant if the systematic uncertainty was also marginalized. The right panel shows the constraints if the true $C_{\ell}$ is used. We also do not see any significant deviation of the constraint from the truth. Both experiments indicate that the errors arising from the imprecisions in PSF modeling and
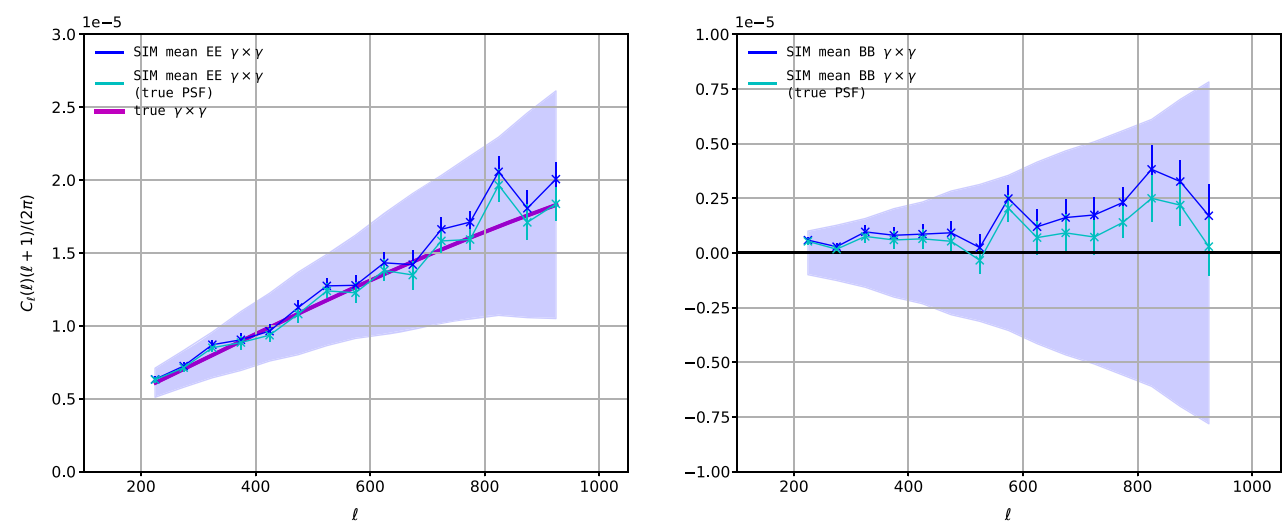

FIG. 13. E-mode (left) and B-mode (right) average power spectra of 30 UFig simulations from the fiducial survey, with different random seeds. The true $C_{\ell}$ is shown with the magenta line. The blue (cyan) line shows the power spectrum calculated using estimated (true) PSF parameters. Error bars on the lines show the error on the mean from 30 power spectra. Light blue bands correspond to $1 \sigma$ errors as calculated from the covariance matrix (see Sec. IV). 

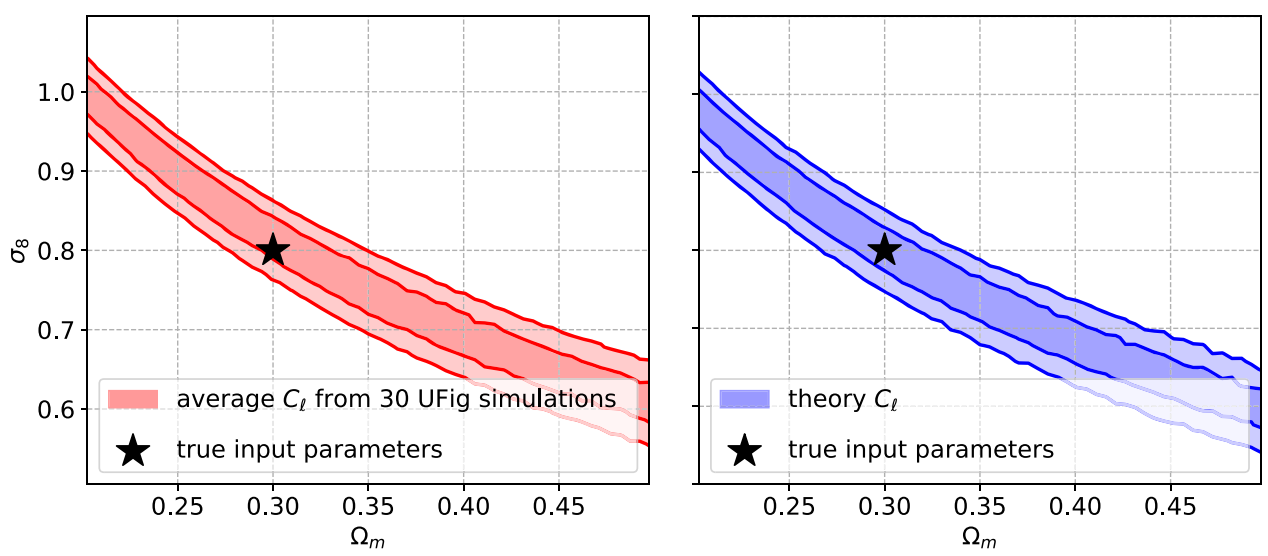

FIG. 14. Constraints from average $C_{\ell}$ from 30 UFig simulations of full Y1 area with different random seeds (left panel). The input cosmology, true shear map, and systematic maps are kept the same. The estimated PSF parameters are used in the $C_{\ell}$ calculation. The true input cosmology is marked with a black star. The right panel shows the constraints obtained when the true $C_{\ell}$ is used as input. The covariance matrix used is the same as in the main analysis. These constraints contain only the statistical uncertainty, no marginalization over systematics from $n(z)$ or shear calibration is performed.

interpolation, as well as finite number of simulations used to create the covariance matrix, are not affecting the constraints on a significant level. We conclude that the analysis pipeline recovers the input cosmological parameters well from internally created simulations.

\section{APPENDIX G: SHEAR 1-PT STATISTICS}

We compare the DES and UFig catalogs in terms of the mean shear of the entire sample as a function of various properties. The mean shear for the full sample is given in Sec. IV D and is much larger than expected from cosmic variance. In our method, the mean shear is subtracted from the maps before power spectrum estimation. This information would not be used anyway, as it contributes only to $\ell<100$. Because of this, we can tolerate mean shear in the data, as long as the 2-pt statistics, and especially the B-mode, remain low. Nevertheless, a statistically significant mean shear would be an indication of possible remaining issues. In Fig. 15 we investigate the behavior of the mean shear as a function of various parameters: PSF shape, size, flexions, position in the survey footprint, brightness and signal-to-noise, colors, and distance to near bright star of magnitude $m<12$, taken from the SKY2000 catalog [82]. We plot only $f_{1}$ and $g_{1}$ flexion, as the other components looked very similar. Colors are plotted only for the DES data, as we simulate the full survey area only for the $i$-band. We plot only the $r-i$ and $g-r$ colors here, other combinations looked similar. We also do not plot dependence on the PSF kurtosis and ration of the Moffat components, as the mean shear as a function of these parameters does not display any trends.

A trend that differs between the DES data and the UFig simulations would be an indication of a remaining issue that was not properly accounted for in the analysis or not modeled correctly in the simulations. We notice a PSF leakage on the level of $\approx 4 \%$ in the DES data. We do not expect it to affect the measurement significantly, as described in Sec. IV A 2. Mean shear in the $\gamma_{1}$ direction is consistently low, and does not seem to depend in a different way than the simulations on any of the variables considered. This suggests that the mean shear behaves purely like a constant offset, which would not have an influence on the shear power spectrum. 

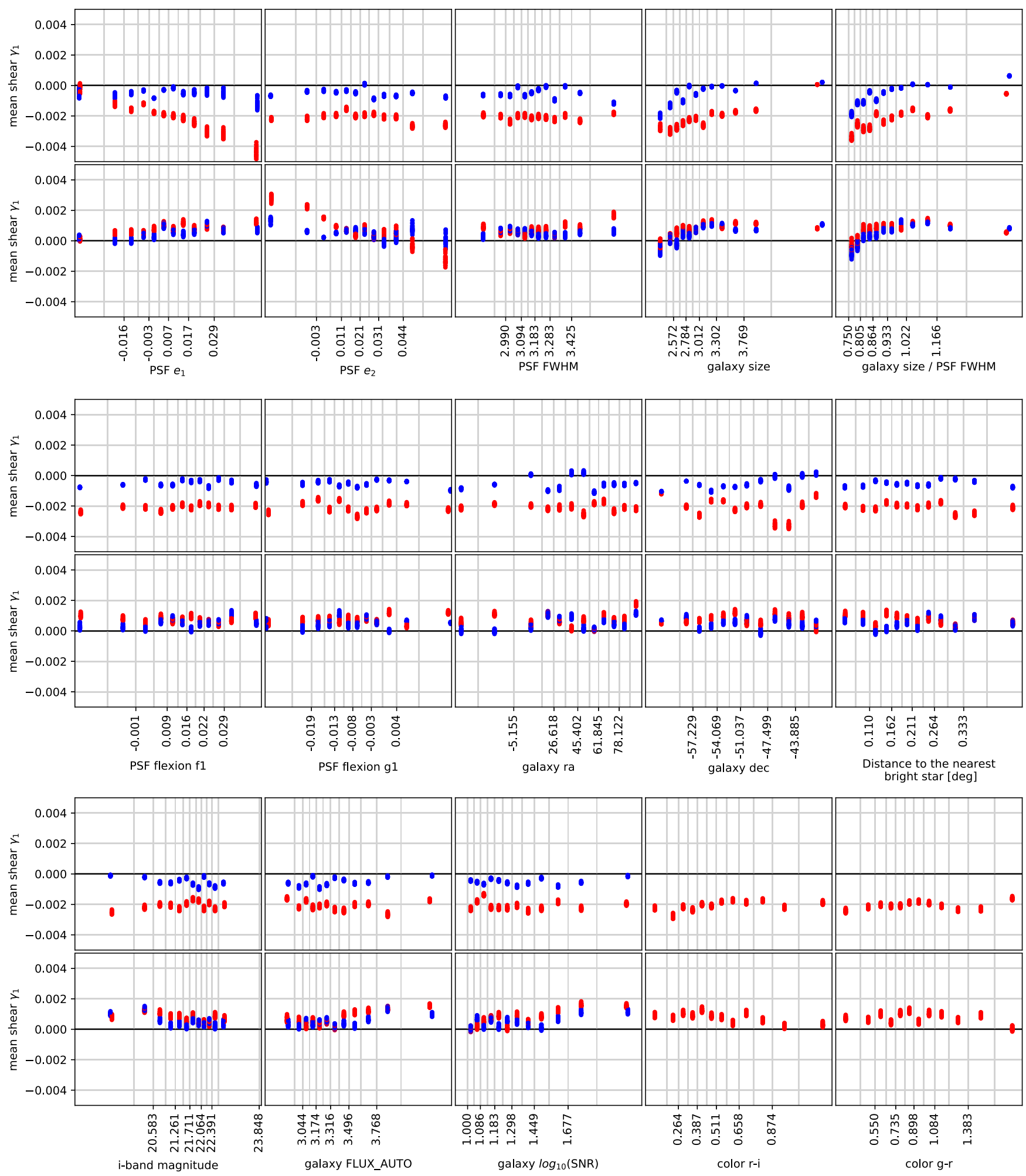

FIG. 15. Mean shear as a function of various parameters. Galaxy size was calculated using SEXTRACTOR weighted moments with Eq. (E1). 


\section{APPENDIX H: NOISE CORRELATION}

The process of image resampling using the Lanczos kernel causes noise to become correlated. We include this correlation in the UFIG simulations by convolving the noise image with a specially designed kernel. We create this kernel such that its autocorrelation is matched to that expected from Lanczos-resampled images. We calculate this expected autocorrelation by measuring the average auto-correlation of a set of 20000 resampled noise images of size $100 \times 100$ pixels. These images are resampled to a shifted coordinate system, with a random uniform shift of maximally \pm 0.5 pixel in both $\mathrm{x}$ and $\mathrm{y}$ directions. The final kernel image calculated this way was contained in $7 \times 7$ pixel stamp, shown in Fig. 16 .

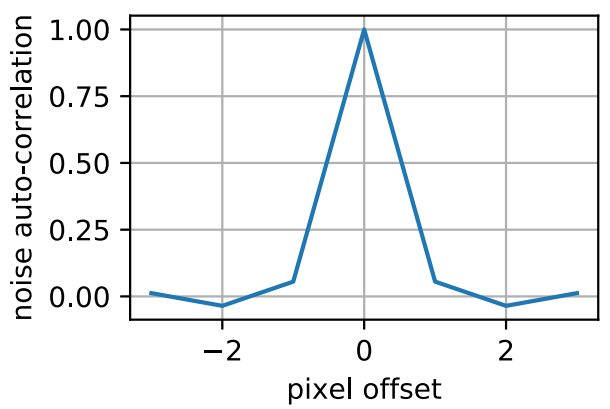

FIG. 16. Convolution kernel used to introduce noise correlation in the simulated images.
[1] D. J. Bacon, A. R. Refregier, and R. S. Ellis, Mon. Not. R. Astron. Soc. 318, 625 (2000).

[2] N. Kaiser, G. Wilson, and G. A. Luppino,arXiv:astro-ph/ 0003338 .

[3] L. Van Waerbeke, Y. Mellier, T. Erben, J. C. Cuillandre, F. Bernardeau, R. Maoli, E. Bertin, H. J. McCracken, O. Le Fèvre, B. Fort, M. Dantel-Fort, B. Jain, and P. Schneider, Astron. Astrophys. 358, 30 (2000).

[4] D. M. Wittman, J. A. Tyson, D. Kirkman, I. Dell'Antonio, and G. Bernstein, Nature (London) 405, 143 (2000).

[5] H. Hildebrandt et al., Astron. Astrophys. 633, A69 (2020).

[6] C. Hikage et al., arXiv:1809.09148.

[7] DES Collaboration, Phys. Rev. D 98, 043526 (2018).

[8] J. Zuntz, T. Kacprzak, L. Voigt, M. Hirsch, B. Rowe, and S. Bridle, Mon. Not. R. Astron. Soc. 434, 1604 (2013).

[9] I. Fenech Conti, R. Herbonnet, H. Hoekstra, J. Merten, L. Miller, and M. Viola, Mon. Not. R. Astron. Soc. 467, 1627 (2017).

[10] E. S. Sheldon and E. M. Huff, Astrophys. J. 841, 24 (2017).

[11] L. Miller et al., Mon. Not. R. Astron. Soc. 429, 2858 (2013).

[12] B. Hoyle et al., Mon. Not. R. Astron. Soc. 478, 592 (2018).

[13] C. Bonnett et al. (Dark Energy Survey Collaboration), Phys. Rev. D 94, 042005 (2016)

[14] M. Bilicki et al., Astron. Astrophys. 616, A69 (2018).

[15] S. Cavuoti, C. Tortora, M. Brescia, G. Longo, M. Radovich, N. R. Napolitano, V. Amaro, C. Vellucci, F. La Barbera, F. Getman, and A. Grado, Mon. Not. R. Astron. Soc. 466, 2039 (2017).

[16] C. Sánchez et al., Mon. Not. R. Astron. Soc. 445, 1482 (2014).

[17] A. Refregier and A. Amara, Phys. Dark Universe 3, 1 (2014).

[18] J. Bergé, L. Gamper, A. Réfrégier, and A. Amara, Astron. Comput. 1, 23 (2013).

[19] C. Bruderer, C. Chang, A. Refregier, A. Amara, J. Bergé, and L. Gamper, Astrophys. J. 817, 25 (2016).

[20] C. Bruderer, A. Nicola, A. Amara, A. Refregier, J. Herbel, and T. Kacprzak,arXiv:1707.06233.
[21] J. Herbel, T. Kacprzak, A. Amara, A. Refregier, C. Bruderer, and A. Nicola, J. Cosmol. Astropart. Phys. 08 (2017) 035.

[22] M. Fagioli, J. Riebartsch, A. Nicola, J. Herbel, A. Amara, A. Refregier, C. Chang, and L. Gamper, arXiv:1803.06343.

[23] L. Tortorelli, L. Della Bruna, J. Herbel, A. Amara, A. Refregier, A. Alarcon, F. J. Castander, J. De Vicente, M. Eriksen, E. Fernandez, J. García-Bellido, E. Gaztanaga, R. Miquel, C. Padilla, E. Sanchez, S. Serrano, L. Stothert, and N. Tonello, arXiv:1805.05340.

[24] G. Bruzual and S. Charlot, Mon. Not. R. Astron. Soc. 344, 1000 (2003).

[25] M. R. Blanton and S. Roweis, Astron. J. 133, 734 (2007).

[26] D. J. Schlegel, D. P. Finkbeiner, and M. Davis, Astrophys. J. 500, 525 (1998).

[27] J. L. Sérsic, Bol. Asoc. Argent. Astron. La Plata Argent. 6, 41 (1963).

[28] F. Tarsitano et al., Mon. Not. R. Astron. Soc. 481, 2018 (2018).

[29] A. Leauthaud et al., Astrophys. J. 744, 159 (2012).

[30] Gaia Collaboration, Astron. Astrophys. 616, A1 (2018).

[31] Http://model.obs-besancon.fr.

[32] A. Robin, C. Reylé, S. Derrière, and S. Picaud, Astron. Astrophys. 409, 523 (2003).

[33] K. M. Gorski, B. D. Wandelt, F. K. Hansen, E. Hivon, and A. J. Banday, arXiv:astro-ph/9905275.

[34] C. Jordi, M. Gebran, J. M. Carrasco, J. de Bruijne, H. Voss, C. Fabricius, J. Knude, A. Vallenari, R. Kohley, and A. Mora, Astron. Astrophys. 523, A48 (2010).

[35] B. Flaugher et al. (DES Collaboration), Astron. J. 150, 150 (2015).

[36] A. Drlica-Wagner et al. (DES Collaboration), Astrophys. J. Suppl. Ser. 235, 33 (2018).

[37] E. Morganson et al. (DES Collaboration), Publ. Astron. Soc. Pac. 130, 074501 (2018).

[38] G. M. Bernstein et al. (DES Collaboration), Publ. Astron. Soc. Pac. 129, 074503 (2017).

[39] E. Bertin and S. Arnouts, Astron. Astrophys. Suppl. Ser. 117, 393 (1996). 
[40] J. Zuntz et al., arXiv:1708.01533.

[41] A. F. J. Moffat, Astron. Astrophys. 3, 455 (1969).

[42] J. Herbel, T. Kacprzak, A. Amara, A. Refregier, and A. Lucchi, J. Cosmol. Astropart. Phys. 07 (2018) 054.

[43] B. Rowe, Mon. Not. R. Astron. Soc. 404, 350 (2010).

[44] M. Sunnåker, A. G. Busetto, E. Numminen, J. Corander, M. Foll, and C. Dessimoz, PLoS Comput. Biol. 9, e1002803 (2013).

[45] J. Akeret, A. Refregier, A. Amara, S. Seehars, and C. Hasner, J. Cosmol. Astropart. Phys. 08 (2015) 043.

[46] O. Le Fèvre et al., Astron. Astrophys. 439, 845 (2005).

[47] B. Garilli et al., Astron. Astrophys. 486, 683 (2008).

[48] O. Le Fèvre et al., Astron. Astrophys. 559, A14 (2013).

[49] I. Szapudi, S. Prunet, D. Pogosyan, A. S. Szalay, and J. R. Bond, Astrophys. J. Lett. 548, L115 (2001).

[50] I. Szapudi, S. Prunet, and S. Colombi, Astrophys. J. Lett. 561, L11 (2001).

[51] G. Chon, A. Challinor, S. Prunet, E. Hivon, and I. Szapudi, Mon. Not. R. Astron. Soc. 350, 914 (2004).

[52] C. Chang, M. Jarvis, B. Jain, S. M. Kahn, D. Kirkby, A. Connolly, S. Krughoff, E. H. Peng, and J. R. Peterson, Mon. Not. R. Astron. Soc. 434, 2121 (2013).

[53] S. Paulin-Henriksson, A. Amara, L. Voigt, A. Refregier, and S. L. Bridle, Astron. Astrophys. 484, 67 (2008).

[54] H. Hildebrandt et al., Mon. Not. R. Astron. Soc. 465, 1454 (2017).

[55] A. Nicola, A. Refregier, and A. Amara, Phys. Rev. D 94, 083517 (2016).

[56] A. Nicola, A. Refregier, and A. Amara, Phys. Rev. D 95, 083523 (2017).

[57] D. N. Limber, Astrophys. J. 117, 134 (1953).

[58] N. Kaiser, Astrophys. J. 388, 272 (1992).

[59] N. Kaiser, Astrophys. J. 498, 26 (1998).

[60] J. A. Peacock, Cosmological Physics, edited by J. A. Peacock (Cambridge University Press, Cambridge, England, 1999), p. 704, ISBN .

[61] A. Refregier, L. Gamper, A. Amara, and L. Heisenberg, arXiv:1708.05177.

[62] D. J. Eisenstein and W. Hu, Astrophys. J. 496, 605 (1998).
[63] A. J. Mead, J. A. Peacock, C. Heymans, S. Joudaki, and A. F. Heavens, Mon. Not. R. Astron. Soc. 454, 1958 (2015).

[64] A. J. Mead, C. Heymans, L. Lombriser, J. A. Peacock, O. I. Steele, and H. A. Winther, Mon. Not. R. Astron. Soc. 459, 1468 (2016).

[65] C. M. Hirata and U. Seljak, Phys. Rev. D 70, 063526 (2004).

[66] Dark Energy Survey Collaboration, Phys. Rev. D 94, 022001 (2016).

[67] C. Heymans et al., Mon. Not. R. Astron. Soc. 432, 2433 (2013).

[68] R. Sgier, A. Refregier, A. Amara, and A. Nicola, arXiv: 1801.05745 .

[69] M. A. Troxel et al., Mon. Not. R. Astron. Soc. 479, 4998 (2018).

[70] C. Howlett, M. Manera, and W. J. Percival, Astron. Comput. 12, 109 (2015).

[71] I. Sobol', USSR Computational Mathematics and Mathematical Physics 7, 86 (1967).

[72] S. Kucherenko, D. Albrecht, and A. Saltelli, arXiv:1505 .02350 .

[73] Planck Collaboration, arXiv:1807.06209.

[74] M. A. Troxel et al., Phys. Rev. D 98, 043528 (2018).

[75] R. E. Smith, J. A. Peacock, A. Jenkins, S. D. M. White, C. S. Frenk, F. R. Pearce, P. A. Thomas, G. Efstathiou, and H. M. P. Couchman, Mon. Not. R. Astron. Soc. 341, 1311 (2003).

[76] R. Takahashi, M. Sato, T. Nishimichi, A. Taruya, and M. Oguri, Astrophys. J. 761, 152 (2012).

[77] Http://www2.iap.fr/users/hivon/software/PolSpice/.

[78] J. Fluri, T. Kacprzak, A. Lucchi, A. Refregier, A. Amara, and T. Hofmann, Phys. Rev. D 98, 123518 (2018).

[79] R. A. Beare, M. J. I. Brown, K. A. Pimbblet, F. Bian, and Y.-T. Lin, Astrophys. J. 815, 94 (2015).

[80] T. Kacprzak, J. Herbel, A. Amara, and A. Réfrégier, arXiv:1707.07498.

[81] A. Gretton, K. M. Borgwardt, M. J. Rasch, A. Schölkopf, and B. Smola, J. Mach. Learn. Res. 13, 723 (2012).

[82] J. R. Myers, C. B. Sande, A. C. Miller, W. H. Warren, Jr., and D. A. Tracewell, VizieR Online Data Catalog 5109 (2001). 